\title{
ZUSGS
}

science for a changing world

\section{Modified Mercalli Intensities For Some California North-Coast Earthquakes}

by William H. Bakun ${ }^{1}$

Open-File Report 99-171

1999

This report is preliminary and has not been reviewed for conformity with U.S. Geological Survey editorial standards or with the North American Stratigraphic Code. Any use of trade, firm, or product names is for descriptive purposes only and does not imply endorsement by the U.S. Government.

U.S. DEPARTMENT OF THE INTERIOR

U.S. GEOLOGICAL SURVEY

${ }^{1}$ Menlo Park, California 


\begin{abstract}
Modified Mercalli Intensity ( $M M I)$ data are used by Bakun (in preparation) to estimate the location and moment magnitude $\mathrm{M}$ of California north-coast earthquakes from MMI observations only. The MMI data and site corrections used by Bakun (in preparation) are listed in this Open-file Report. This report is also accessible at http://quake.wr.usgs.gov/ bakun/.
\end{abstract}

\title{
Explanation of Table 1
}

Intensity observations have been obtained from several sources that assign intensity values according to descriptions in the Modified Mercalli Intensity ( $M M I)$ scale proposed by Wood and Neumann (1931): Toppozada et al. (1981) for earthquakes before 1900, Toppozada et al. (1982) for the 29 October 1909 and 22 January 1923 earthquakes, Townley and Allen (1939) for the 31 January 1922 shock, United States Earthquakes for earthquakes in the interval 1931-1986 [Neumann (1934, 1936, 1938, 1943), Bodle and Murphy (1947), Murphy and Cloud (1953, 1956), Brazee and Cloud (1958), Talley and Cloud (1962), Lander and Cloud (1964), Stover and von Hake (1982), and Stover and Brewer (1994)], and Dreger et al. (1995) for the 17 August 1991 earthquake.

Unpublished intensity data of the U S Geological Survey (J. W. Dewey, written communication, 1997) were used for the 31 July 1987, 25 April 1992, and 1 September 1994 earthquakes.

The Modified Mercalli observations (MMI) in Table 1 are listed in chronological order, then by decreasing MMI, and finally by community name in alphabetical order. The site corrections listed for 8 sites for the 15 April 1898 earthquake were determined by Bakun and Wentworth (1997). 


\section{References}

Bakun, W. H. (in preparation). Seismicity of California's north coast.

Bakun, W. H. and C. M. Wentworth (1997). Estimating earthquake location and magnitude from seismic intensity data, Bull. Seismol. Soc. Amer. 87, 1502-1521.

Bodle, R. R., and L. M. Murphy (1947). United States Earthquakes 1945, U.S. Coast Geodetic Survey, Washington, D.C., 38pp.

Brazee, R. J., and W. K. Cloud (1958). United States Earthquakes 1956, U.S. Coast Geodetic Survey, Washington, D.C., 77pp.

Dreger, D. S., L. S. Gee, S. Loper, M. R. McKenzie, and R. A. Uhrhammer (1995). Bulletin of the Seismographic Stations of the University of California, Volumes 60 and 61 January 1, 1990 to December 31, 1991, 79pp.

Lander. J. F., and W. K. Cloud (1964). United States Earthquakes 1962, U.S. Coast Geodetic Survey, Washington, D.C., 114pp.

Murphy, L. M., and W. K. Cloud (1953). United States Earthquakes 1951, U.S. Coast Geodetic Survey, Washington, D.C., 49pp.

Murphy, L. M., and W. K. Cloud (1956). United States Earthquakes 1954, U.S. Coast Geodetic Survey, Washington, D.C., 110pp.

Neumann, F. (1934). United States Earthquakes 1932, U.S. Coast Geodetic Survey, Washington, D.C., $21 \mathrm{pp}$.

Neumann, F. (1936). United States Earthquakes 1934, U.S. Coast Geodetic Survey, Washington, D.C., $101 \mathrm{pp}$.

Neumann, F. (1938). United States Earthquakes 1936, U.S. Coast Geodetic Survey, Washington, D.C., 44pp.

Neumann, F. (1943). United States Earthquakes 1941, U.S. Coast Geodetic Survey, Washington, D.C., 68pp.

Stover, C. W. and L. R. Brewer (1994). United States Earthquakes, 1986, U.S. Geological Survey Bulletin 2089, 240pp.

Stover, C. W. and J. L. Coffman (1993). Seismicity of the United States, 1568-1989 (Revised), U. S. Geological Survey Prof. Pap. 1527, 418pp.

$$
\text { iL }
$$


Talley, Jr., H. C. and W. K. Cloud (1962). United States Earthquakes 1960, U.S. Coast Geodetic Survey, Washington, D.C., 90pp.

Toppozada, T. R., and D. L. Parke (1982). Areas damaged by California earthquakes, 1900-1949, Calif. Div. Mines and Geol. Open-file Rept. 82-17 SAC, 65 pp.

Toppozada, T. R., C. R. Real and D. L. Parke (1981). Preparation of isoseismal maps and summaries of reported effects for pre-1900 California earthquakes, Calif. Div. Mines and Geol. Open-file Rept. 81-11 SAC,182 pp

Townley, S. D., and M. W. Allen (1939). Descriptive catalog of earthquakes of the Pacific Coast of the United States 1769 to 1928, Bull. Seismol. Soc. Am., 29, 297pp.

Wood, H. O., and F. Neumann (1931). Modified Mercalli Intensity Scale of 1931, Bull. Seismol. Soc. Am., 21, pp. 277-283. 
Table 1. Modified Mercalli Intensity Observations for California North-Coast Earthquakes

\begin{tabular}{|c|c|c|c|c|c|}
\hline Mo-Day-Yr & Location & St & MMI & Long $\left({ }^{\circ} \mathrm{W}\right)$ & Lat $\left({ }^{\circ} \mathbf{N}\right)$ \\
\hline $10-23-1853$ & Eureka & $\mathrm{CA}$ & 6 & 124.163 & 40.802 \\
\hline $11-13-1860$ & Eureka & $\mathrm{CA}$ & 7 & 124.163 & 40.802 \\
\hline $10-1-1865$ & Eureka & $\mathrm{CA}$ & 7 & 124.163 & 40.802 \\
\hline $10-1-1865$ & Fort Humboldt & $\mathrm{CA}$ & 7 & 124.188 & 40.777 \\
\hline $3-2-1871$ & Mattole & $\mathrm{CA}$ & 8 & 124.284 & 40.322 \\
\hline $3-2-1871$ & Bucksport & CA & 7 & 124.191 & 40.775 \\
\hline $3-2-1871$ & Cape Mendocino Light House & $\mathrm{CA}$ & 7 & 124.406 & 40.440 \\
\hline $3-2-1871$ & Eureka & $\mathrm{CA}$ & 7 & 124.163 & 40.802 \\
\hline $3-2-1871$ & Hydesville & CA & 7 & 124.096 & 40.548 \\
\hline $3-2-1871$ & Rohnerville & $\mathrm{CA}$ & 7 & 124.134 & 40.567 \\
\hline $3-2-1871$ & Centerville & CA & 6 & 124.347 & 40.575 \\
\hline $3-2-1871$ & Weaverville & CA & 4 & 122.941 & 40.731 \\
\hline $3-2-1871$ & Caparum & $\mathrm{CA}$ & 3 & 123.540 & 41.250 \\
\hline $3-2-1871$ & Orleans & $\mathrm{CA}$ & 3 & 123.540 & 41.302 \\
\hline $11-23-1873$ & Smith River Valley & CA & 8 & 124.146 & 41.928 \\
\hline $11-23-1873$ & Crescent City & $\mathrm{CA}$ & 7 & 124.201 & 41.756 \\
\hline $11-23-1873$ & Grants Pass & CR & 7 & 123.327 & 42.439 \\
\hline $11-23-1873$ & Jacksonville & CR & 7 & 122.966 & 42.314 \\
\hline $11-23-1873$ & Weaverville & $\mathrm{CA}$ & 6 & 122.941 & 40.731 \\
\hline $11-23-1873$ & Eureka & $\mathrm{CA}$ & 5.5 & 124.163 & 40.802 \\
\hline $11-23-1873$ & Indian Creek & CA & 5.5 & 122.913 & 40.658 \\
\hline $11-23-1873$ & Red Bluff & CA & 5.5 & 122.235 & 40.179 \\
\hline $11-23-1873$ & Scott Bar & $\mathrm{CA}$ & 5.5 & 123.003 & 41.742 \\
\hline $11-23-1873$ & Scott Valley & $\mathrm{CA}$ & 5.5 & 122.855 & 41.601 \\
\hline $11-23-1873$ & Yreka & $\mathrm{CA}$ & 5.5 & 122.633 & 41.736 \\
\hline $11-23-1873$ & Bogus & $\mathrm{CA}$ & 5 & 122.363 & 41.927 \\
\hline $11-23-1873$ & Sacramento & CA & 5 & 121.493 & 38.582 \\
\hline $11-23-1873$ & Ashland & CR & 4.5 & 122.708 & 42.195 \\
\hline $11-23-1873$ & Cottonwood & CA & 4.5 & 122.280 & 40.386 \\
\hline $11-23-1873$ & Roseburg & OR & 4.5 & 123.341 & 43.217 \\
\hline $9-30-1875$ & Eureka & $\mathrm{CA}$ & 7 & 124.163 & 40.802 \\
\hline $9-30-1875$ & Arcata & $\mathrm{CA}$ & 5 & 124.082 & 40.867 \\
\hline $9-30-1875$ & Weaverville & $\mathrm{CA}$ & 5 & 122.941 & 40.731 \\
\hline $5-9-1878$ & Petrolia & $\mathrm{CA}$ & 7 & 124.286 & 40.326 \\
\hline $5-9-1878$ & Big Flat & $\mathrm{CA}$ & 6 & 124.183 & 40.132 \\
\hline $5-9-1878$ & Grizzly Bluff & $\mathrm{CA}$ & 6 & 124.170 & 40.562 \\
\hline $5-9-1878$ & Albion & CA & 5.5 & 123.768 & 39.224 \\
\hline $5-9-1878$ & Bear River & $\mathrm{CA}$ & 5.5 & 124.389 & 40.477 \\
\hline $5-9-1878$ & Kibesillah & $\mathrm{CA}$ & 5.5 & 123.777 & 39.590 \\
\hline $5-9-1878$ & Eureka & $\mathrm{CA}$ & 5 & 124.163 & 40.802 \\
\hline $5-9-1878$ & Ferndale & $\mathrm{CA}$ & 5 & 124.263 & 40.576 \\
\hline $5-9-1878$ & Point Arena & $\mathrm{CA}$ & 5 & 123.692 & 38.909 \\
\hline $5-9-1878$ & Red Bluff & CA & 5 & 122.235 & 40.179 \\
\hline $5-9-1878$ & Sacramento & $\mathrm{CA}$ & 5 & 121.493 & 38.582 \\
\hline 5-9-1878 & Shelter Cove & $\mathrm{CA}$ & 5 & 124.072 & 40.031 \\
\hline $5-9-1878$ & Ukiah & CA & 5 & 123.207 & 39.150 \\
\hline
\end{tabular}


Table 1. Modified Mercalli Intensity Observations for California North-Coast

Earthquakes

\begin{tabular}{|c|c|c|c|c|c|c|c|}
\hline Mo-Day-Yr & Location & St & MMl & Long $\left({ }^{\circ} \mathrm{W}\right)$ & Lat $\left({ }^{\circ} \mathbf{N}\right)$ & Site Corr & $\begin{array}{l}\text { MMI- } \\
\text { Site } \\
\text { Corr }\end{array}$ \\
\hline 5-9-1878 & Williams & $\mathrm{CA}$ & 5 & 122.148 & 39.155 & & \\
\hline 5-9-1878 & Casper & $\mathrm{CA}$ & 4.5 & 123.815 & 39.364 & & \\
\hline $5-9-1878$ & Cuffey's Cove & $\mathrm{CA}$ & 4.5 & 123.730 & 39.141 & & \\
\hline $5-9-1878$ & Mendocino & $\mathrm{CA}$ & 4.5 & 123.798 & 39.308 & & \\
\hline 5-9-1878 & Westport & $\mathrm{CA}$ & 4.5 & 123.782 & 39.636 & & \\
\hline $5-9-1878$ & Lakeport & $\mathrm{CA}$ & 4 & 122.915 & 39.043 & & \\
\hline $1-28-1884$ & Fort Jones & $\mathrm{CA}$ & 5 & 122.839 & 41.608 & & \\
\hline $7-26-1890$ & Grizzly Bluff & CA & 7 & 124.170 & 40.562 & & \\
\hline $7-26-1890$ & Petrolia & CA & 7 & 124.286 & 40.326 & & \\
\hline $7-26-1890$ & Arcata & CA & 5.5 & 124.082 & 40.867 & & \\
\hline $7-26-1890$ & Crescent City & $\mathrm{CA}$ & 5 & 124.201 & 41.756 & & \\
\hline $7-26-1890$ & Eureka & $\mathrm{CA}$ & 5 & 124.163 & 40.802 & & \\
\hline $7-26-1890$ & Mendocino & CA & 5 & 123.798 & 39.308 & & \\
\hline $7-26-1890$ & Weaverville & $\mathrm{CA}$ & 4 & 122.941 & 40.731 & & \\
\hline $9-30-1894$ & South Humboldt Co. & CA & 7 & 124.200 & 40.200 & & \\
\hline $9-30-1894$ & Eureka & $C A$ & 5 & 124.163 & 40.802 & & \\
\hline $9-30-1894$ & Shasta & CA & 5 & 122.491 & 40.599 & & \\
\hline $9-30-1894$ & Long Ridge & $\mathrm{CA}$ & 4 & 123.333 & 40.076 & & \\
\hline $4-15-1898$ & Greenwood & $\mathrm{CA}$ & 9 & 123.718 & 39.135 & & \\
\hline $4-15-1898$ & Noyo & CA & 9 & 123.802 & 39.428 & & \\
\hline $4-15-1898$ & Little River & CA & 8 & 123.787 & 39.271 & & \\
\hline $4-15-1898$ & Mendocino & $\mathrm{CA}$ & 8 & 123.798 & 39.308 & & \\
\hline $4-15-1898$ & Comptche & $\mathrm{CA}$ & 7 & 123.590 & 39.265 & & \\
\hline $4-15-1898$ & Fort Bragg & $\mathrm{CA}$ & 7 & 123.804 & 39.446 & & \\
\hline $4-15-1898$ & Pine Grove & $\mathrm{CA}$ & 7 & 123.813 & 39.349 & & \\
\hline $4-15-1898$ & Ukiah & $\mathrm{CA}$ & 7 & 123.207 & 39.150 & 1.19 & 5.81 \\
\hline $4-15-1898$ & Albion & $\mathrm{CA}$ & 6 & 123.768 & 39.224 & & \\
\hline $4-15-1898$ & Point Arena & $\mathrm{CA}$ & 6 & 123.692 & 38.909 & & \\
\hline $4-15-1898$ & Berkeley & $\mathrm{CA}$ & 5 & 122.272 & 37.872 & -0.02 & 5.02 \\
\hline $4-15-1898$ & Eureka & CA & 5 & 124.163 & 40.802 & & \\
\hline $4-15-1898$ & Red Bluff & $\mathrm{CA}$ & 5 & 122.235 & 40.179 & 0.36 & 4.64 \\
\hline $4-15-1898$ & San Francisco & $\mathrm{CA}$ & 5 & 122.418 & 37.775 & 1.18 & 3.82 \\
\hline $4-15-1898$ & Santa Rosa & $\mathrm{CA}$ & 5 & 122.713 & 38.441 & 0.41 & 4.59 \\
\hline 4-15-1898 & Willows & $\mathrm{CA}$ & 5 & 122.193 & 39.524 & -0.54 & 5.54 \\
\hline $4-15-1898$ & Lytton Springs & $\mathrm{CA}$ & 4.5 & 122.871 & 38.659 & & 4.50 \\
\hline $4-15-1898$ & Oakland & $\mathrm{CA}$ & 4.5 & 122.270 & 37.804 & -0.14 & 4.64 \\
\hline $4-15-1898$ & Port Costa & $\mathrm{CA}$ & 4.5 & 122.182 & 38.046 & -0.33 & 4.83 \\
\hline $4-16-1899$ & Eureka & $\mathrm{CA}$ & 6 & 124.163 & 40.802 & & \\
\hline 4-16-1899 & Arcata & $\mathrm{CA}$ & 5 & 124.082 & 40.867 & & \\
\hline $4-16-1899$ & Crescent City & $\mathrm{CA}$ & 5 & 124.201 & 41.756 & & \\
\hline $4-16-1899$ & Ferndale & $\mathrm{CA}$ & 5 & 124.263 & 40.576 & & \\
\hline 10-29-1909 & Newburg & $\mathrm{CA}$ & 8 & 124.124 & 40.600 & & \\
\hline 10-29-1909 & Rio Dell & $\mathrm{CA}$ & 8 & 124.105 & 40.499 & & \\
\hline 10-29-1909 & Rohnerville & $\mathrm{CA}$ & 8 & 124.134 & 40.567 & & \\
\hline 10-29-1909 & Fortuna & $\mathrm{CA}$ & 7.5 & 124.156 & 40.598 & & \\
\hline $10-29-1909$ & Upper Mattole & $\mathrm{CA}$ & 7.5 & 124.165 & 40.252 & & \\
\hline
\end{tabular}


Table 1. Modified Mercalli Intensity Observations for California North-Coast Earthquakes

\begin{tabular}{|c|c|c|c|c|c|}
\hline Mo-Day-Yr & Location & St & MMI & Long $\left({ }^{\circ} W\right)$ & $\operatorname{Lat}\left({ }^{\circ} \mathbf{N}\right)$ \\
\hline $10-29-1909$ & Eureka & $\mathrm{CA}$ & 7 & 124.163 & 40.802 \\
\hline 10-29-1909 & Ferndale & $\mathrm{CA}$ & 7 & 124.263 & 40.576 \\
\hline 10-29-1909 & Hayfork & $\mathrm{CA}$ & 7 & 123.182 & 40.554 \\
\hline 10-29-1909 & Scotia & $\mathrm{CA}$ & 7 & 124.100 & 40.483 \\
\hline $10-29-1909$ & Blocksburg & $\mathrm{CA}$ & 5 & 123.635 & 40.276 \\
\hline 10-29-1909 & Crescent City & $\mathrm{CA}$ & 5 & 124.201 & 41.756 \\
\hline 10-29-1909 & Redding & $\mathrm{CA}$ & 5 & 122.391 & 40.587 \\
\hline 10-29-1909 & Sisson & $\mathrm{CA}$ & 5 & 122.309 & 41.310 \\
\hline $10-29-1909$ & Willows & $\mathrm{CA}$ & 5 & 122.193 & 39.524 \\
\hline $10-29-1909$ & Grass Valley & $\mathrm{CA}$ & 4 & 121.060 & 39.219 \\
\hline 10-29-1909 & Mendocino & CA & 4 & 123.798 & 39.308 \\
\hline 10-29-1909 & Weaverville & CA & 4 & 122.941 & 40.731 \\
\hline 10-29-1909 & Chico & $\mathrm{CA}$ & 3 & 121.836 & 39.729 \\
\hline $1-31-1922$ & Eureka & $\mathrm{CA}$ & 5 & 124.163 & 40.802 \\
\hline $1-31-1922$ & Red Bluff & $\mathrm{CA}$ & 4 & 122.235 & 40.179 \\
\hline $1-31-1922$ & San Francisco & CA & 3 & 122.418 & 37.775 \\
\hline $1-31-1922$ & Roseburg & CR & 5 & 123.341 & 43.217 \\
\hline 1-31-1922 & Eugene & CR & 3 & 123.086 & 44.052 \\
\hline $1-22-1923$ & Ferndale & CA & 8 & 124.263 & 40.576 \\
\hline $1-22-1923$ & Pepperwood & $\mathrm{CA}$ & 8 & 123.992 & 40.446 \\
\hline $1-22-1923$ & Petrolia & CA & 8 & 124.286 & 40.326 \\
\hline $1-22-1923$ & Scotia & CA & 7.5 & 124.100 & 40.483 \\
\hline $1-22-1923$ & Upper Mattole & CA & 7.5 & 124.165 & 40.252 \\
\hline $1-22-1923$ & Alton & CA & 7 & 124.140 & 40.548 \\
\hline $1-22-1923$ & Dyerville & $\mathrm{CA}$ & 7 & 123.925 & 40.356 \\
\hline $1-22-1923$ & Fortuna & CA & 7 & 124.156 & 40.598 \\
\hline $1-22-1923$ & Loleta & $\mathrm{CA}$ & 7 & 124.224 & 40.641 \\
\hline $1-22-1923$ & Ocean House & $\mathrm{CA}$ & 7 & 124.398 & 40.431 \\
\hline $1-22-1923$ & Arcata & $\mathrm{CA}$ & 6 & 124.082 & 40.867 \\
\hline $1-22-1923$ & Carlotta & CA & 5 & 124.059 & 40.538 \\
\hline $1-22-1923$ & Chico & $\mathrm{CA}$ & 5 & 121.836 & 39.729 \\
\hline $1-22-1923$ & Corning & CA & 5 & 122.178 & 39.928 \\
\hline $1-22-1923$ & Kennett & $\mathrm{CA}$ & 5 & 122.406 & 40.742 \\
\hline $1-22-1923$ & Los Molinos & $\mathrm{CA}$ & 5 & 122.099 & 40.021 \\
\hline $1-22-1923$ & Meridian & $\mathrm{CA}$ & 5 & 121.913 & 39.143 \\
\hline $1-22-1923$ & Montgomery Creek & $\mathrm{CA}$ & 5 & 121.923 & 40.842 \\
\hline $1-22-1923$ & Red Bluff & CA & 5 & 122.235 & 40.179 \\
\hline $1-22-1923$ & Redding & $\mathrm{CA}$ & 5 & 122.391 & 40.587 \\
\hline $1-22-1923$ & San Francisco & CA & 5 & 122.418 & 37.775 \\
\hline $1-22-1923$ & Santa Rosa & CA & 5 & 122.713 & 38.441 \\
\hline $1-22-1923$ & Ukiah & CA & 5 & 123.207 & 39.150 \\
\hline $1-22-1923$ & Willows & $\mathrm{CA}$ & 5 & 122.193 & 39.524 \\
\hline $1-22-1923$ & Sacramento & $\mathrm{CA}$ & 4.5 & 121.493 & 38.582 \\
\hline $1-22-1923$ & Cecilville & $\mathrm{CA}$ & 4 & 123.139 & 41.141 \\
\hline $1-22-1923$ & Sisson & CA & 4 & 122.309 & 41.310 \\
\hline $1-22-1923$ & Yreka & $\mathrm{CA}$ & 4 & 122.633 & 41.736 \\
\hline
\end{tabular}


Table 1. Modified Mercalli Intensity Observations for California North-Coast Earthquakes

\begin{tabular}{|c|c|}
\hline Mo-Day-Yr & Location \\
\hline $6-6-1932$ & Arcata \\
\hline $6-6-1932$ & Bayside \\
\hline $6-6-1932$ & Beatrice \\
\hline $6-6-1932$ & Eureka \\
\hline $6-6-1932$ & Fields Landing \\
\hline $6-6-1932$ & Loleta \\
\hline $6-6-1932$ & Samoa \\
\hline $6-6-1932$ & Blue Lake \\
\hline $6-6-1932$ & Ferndale \\
\hline $6-6-1932$ & Kneeland \\
\hline $6-6-1932$ & Bridgeville \\
\hline $6-6-1932$ & Crannell \\
\hline $6-6-1932$ & Crescent City \\
\hline $6-6-1932$ & Fort Jones \\
\hline $6-6-1932$ & Fortuna \\
\hline $6-6-1932$ & Korbel \\
\hline $6-6-1932$ & Alderpoint \\
\hline $6-6-1932$ & Alton \\
\hline $6-6-1932$ & Ashland \\
\hline $6-6-1932$ & Benbow \\
\hline $6-6-1932$ & Bieber \\
\hline $6-6-1932$ & Capetown \\
\hline $6-6-1932$ & Dyerville \\
\hline $6-6-1932$ & Fernbridge \\
\hline $6-6-1932$ & Forks Of Salmon \\
\hline $6-6-1932$ & Fort Dick \\
\hline $6-6-1932$ & Garberville \\
\hline $6-6-1932$ & Hydesviile \\
\hline $6-6-1932$ & Little River \\
\hline $6-6-1932$ & Longvale \\
\hline $6-6-1932$ & Montgomery Creek \\
\hline $6-6-1932$ & Mount Shasta \\
\hline $6-6-1932$ & Orick \\
\hline $6-6-1932$ & Round Mountain \\
\hline $6-6-1932$ & Scotia \\
\hline $6-6-1932$ & Shively \\
\hline $6-6-1932$ & Somes Bar \\
\hline $6-6-1932$ & Upper Mattole \\
\hline $6-6-1932$ & Weitchpec \\
\hline $6-6-1932$ & Westport \\
\hline $6-6-1932$ & Willow Creek \\
\hline $6-6-1932$ & Anderson \\
\hline $6-6-1932$ & Baird \\
\hline $6-6-1932$ & Bayles \\
\hline $6-6-1932$ & Black Bear \\
\hline $6-6-1932$ & Blocksburg \\
\hline
\end{tabular}

$\begin{array}{llll}\text { St MMI Long }\left({ }^{\circ} \mathrm{W}\right) & \text { Lat }\left({ }^{\circ} \mathrm{N}\right) \quad \text { Site Corr } \quad \begin{array}{c}\text { MMl- } \\ \text { Site } \\ \text { Corr }\end{array}\end{array}$

$\begin{array}{llll}\mathrm{CA} & 8 & 124.082 & 40.867 \\ \mathrm{CA} & 8 & 124.063 & 40.843 \\ \mathrm{CA} & 8 & 124.201 & 40.670 \\ \mathrm{CA} & 8 & 124.163 & 40.802\end{array}$

$\begin{array}{llll}\text { CA } & 8 & 124.214 & 40.725\end{array}$

$\begin{array}{llll}\text { CA } & 8 & 124.224 & 40.641\end{array}$

$\begin{array}{llll}\text { CA } & 8 & 124.185 & 40.819\end{array}$

$\begin{array}{llll}\text { CA } & 7 & 123.983 & 40.883\end{array}$

$\begin{array}{llll}\text { CA } & 7 & 124.263 & 40.576\end{array}$

$\begin{array}{llll}\text { CA } & 7 & 123.994 & 40.761\end{array}$

$\begin{array}{llll}\text { CA } & 6 & 123.799 & 40.469\end{array}$

$\begin{array}{llll}C A & 6 & 124.084 & 41.012\end{array}$

$\begin{array}{llll}\text { CA } & 6 & 124.201 & 41.756\end{array}$

$\begin{array}{llll}C A & 6 & 122.839 & 41.608\end{array}$

$\begin{array}{llll}C A & 6 & 124.156 & 40.598\end{array}$

$\begin{array}{llll}C A & 6 & 123.957 & 40.871\end{array}$

$\begin{array}{llll}C A & 5 & 123.611 & 40.176\end{array}$

$\begin{array}{llll}C A & 5 & 124.140 & 40.548\end{array}$

$\begin{array}{llll}C A & 5 & 122.708 & 42.195\end{array}$

$\begin{array}{llll}C A & 5 & 123.783 & 40.069\end{array}$

$\begin{array}{llll}C A & 5 & 121.143 & 41.121\end{array}$

$\begin{array}{llll}\text { CA } & 5 & 124.366 & 40.466\end{array}$

$\begin{array}{llll}C A & 5 & 123.925 & 40.356\end{array}$

$\begin{array}{llll}\text { CA } & 5 & 124.200 & 40.616\end{array}$

$\begin{array}{llll}C A & 5 & 123.330 & 41.260\end{array}$

$\begin{array}{llll}C A & 5 & 124.148 & 41.868\end{array}$

$\begin{array}{llll}C A & 5 & 123.794 & 40.100\end{array}$

$\begin{array}{llll}C A & 5 & 124.096 & 40.548\end{array}$

$\begin{array}{llll}\text { CA } & 5 & 123.787 & 39.271\end{array}$

$\begin{array}{llll}\text { CA } & 5 & 123.429 & 39.555\end{array}$

$\begin{array}{llll}C A & 5 & 121.923 & 40.842\end{array}$

$\begin{array}{llll}\text { CA } & 5 & 122.309 & 41.310\end{array}$

$\begin{array}{llll}\text { CA } & 5 & 124.059 & 41.287\end{array}$

$\begin{array}{llll}\text { CA } & 5 & 121.941 & 40.794\end{array}$

$\begin{array}{llll}\text { CA } & 5 & 124.100 & 40.483\end{array}$

$\begin{array}{llll}\text { CA } & 5 & 123.969 & 40.431\end{array}$

$\begin{array}{llll}\text { CA } & 5 & 123.475 & 41.376\end{array}$

$\begin{array}{llll}\mathrm{CA} & 5 & 124.165 & 40.252\end{array}$

$\begin{array}{llll}\text { CA } & 5 & 123.707 & 41.188\end{array}$

$\begin{array}{llll}\mathrm{CA} & 5 & 123.782 & 39.636\end{array}$

$\begin{array}{llll}C A & 5 & 123.630 & 40.940\end{array}$

$\begin{array}{llll}\text { CA } & 4 & 122.297 & 40.448\end{array}$

$\begin{array}{llll}C A & 4 & 122.295 & 40.793\end{array}$

$\begin{array}{llll}C A & 4 & 122.641 & 40.149\end{array}$

$\begin{array}{llll}\text { CA } & 4 & 123.180 & 41.240\end{array}$

$\begin{array}{llll}\text { CA } & 4 & 123.635 & 40.276\end{array}$ 
Table 1. Modified Mercalli Intensity Observations for California North-Coast Earthquakes

\begin{tabular}{|c|c|c|c|c|c|}
\hline Mo-Day-Yr & Location & St & MMI & Long $\left({ }^{\circ} W\right)$ & $\operatorname{Lat}\left({ }^{\circ} \mathbf{N}\right)$ \\
\hline $6-6-1932$ & Briceland & $\mathrm{CA}$ & 4 & 123.899 & 40.108 \\
\hline $6-6-1932$ & Burney & $\mathrm{CA}$ & 4 & 121.660 & 40.883 \\
\hline $6-6-1932$ & Carrville & $\mathrm{CA}$ & 4 & 122.703 & 41.065 \\
\hline $6-6-1932$ & Castella & CA & 4 & 122.317 & 41.139 \\
\hline $6-6-1932$ & Cecilville & $\mathrm{CA}$ & 4 & 123.139 & 41.141 \\
\hline $6-6-1932$ & Chico & CA & 4 & 121.836 & 39.729 \\
\hline $6-6-1932$ & Clipper Mills & $\mathrm{CA}$ & 4 & 121.156 & 39.533 \\
\hline $6-6-1932$ & Colusa & $\mathrm{CA}$ & 4 & 122.008 & 39.214 \\
\hline $6-6-1932$ & Copco & $\mathrm{CA}$ & 4 & 122.360 & 41.983 \\
\hline $6-6-1932$ & Cottonwood & CA & 4 & 122.280 & 40.386 \\
\hline $6-6-1932$ & Dedrick & $\mathrm{CA}$ & 4 & 123.036 & 40.863 \\
\hline $6-6-1932$ & Del Loma & $\mathrm{CA}$ & 4 & 123.331 & 40.779 \\
\hline $6-6-1932$ & Dos Rios & $\mathrm{CA}$ & 4 & 123.352 & 39.717 \\
\hline $6-6-1932$ & Dunsmuir & $\mathrm{CA}$ & 4 & 122.271 & 41.208 \\
\hline $6-6-1932$ & Durham & $\mathrm{CA}$ & 4 & 121.799 & 39.646 \\
\hline $6-6-1932$ & Edgewood & $\mathrm{CA}$ & 4 & 122.431 & 41.458 \\
\hline $6-6-1932$ & Etna & CA & 4 & 122.894 & 41.457 \\
\hline $6-6-1932$ & Ettersburg & $\mathrm{CA}$ & 4 & 123.996 & 40.139 \\
\hline $6-6-1932$ & Fort Bragg & $\mathrm{CA}$ & 4 & 123.804 & 39.446 \\
\hline $6-6-1932$ & French Gulch & $\mathrm{CA}$ & 4 & 122.637 & 40.701 \\
\hline $6-6-1932$ & Grass Valley & $\mathrm{CA}$ & 4 & 121.060 & 39.219 \\
\hline $6-6-1932$ & Greenville & $\mathrm{CA}$ & 4 & 120.950 & 40.140 \\
\hline $6-6-1932$ & Happy Camp & $\mathrm{CA}$ & 4 & 123.378 & 41.793 \\
\hline $6-6-1932$ & Harris & $\mathrm{CA}$ & 4 & 123.658 & 40.084 \\
\hline $6-6-1932$ & Hartsook & $\mathrm{CA}$ & 4 & 123.790 & 40.010 \\
\hline $6-6-1932$ & Hoopa & $\mathrm{CA}$ & 4 & 123.673 & 41.051 \\
\hline $6-6-1932$ & Horse Creek & $\mathrm{CA}$ & 4 & 122.996 & 41.824 \\
\hline $6-6-1932$ & Hyampom & CA & 4 & 123.451 & 40.618 \\
\hline $6-6-1932$ & Junction City & $\mathrm{CA}$ & 4 & 123.053 & 40.733 \\
\hline $6-6-1932$ & Mendocino & $\mathrm{CA}$ & 4 & 123.798 & 39.308 \\
\hline 6-6-1932 & Millville & CA & 4 & 122.174 & 40.549 \\
\hline $6-6-1932$ & Mina & $\mathrm{CA}$ & 4 & 123.357 & 39.965 \\
\hline $6-6-1932$ & Minersville & CA & 4 & 122.782 & 40.851 \\
\hline $6-6-1932$ & Miranda & CA & 4 & 123.823 & 40.235 \\
\hline $6-6-1932$ & Montague & $\mathrm{CA}$ & 4 & 122.527 & 41.728 \\
\hline $6-6-1932$ & Nevada City & CA & 4 & 121.015 & 39.262 \\
\hline $6-6-1932$ & Palo Cedro & CA & 4 & 122.238 & 40.564 \\
\hline $6-6-1932$ & Paradise & CA & 4 & 121.621 & 39.760 \\
\hline $6-6-1932$ & Peanut & CA & 4 & 123.168 & 40.468 \\
\hline $6-6-1932$ & Piercy & CA & 4 & 123.794 & 39.966 \\
\hline $6-6-1932$ & Point Cabrillo Light Station & CA & 4 & 123.826 & 39.349 \\
\hline $6-6-1932$ & Pollock & CA & 4 & 122.385 & 40.917 \\
\hline $6-6-1932$ & Redding & CA & 4 & 122.391 & 40.587 \\
\hline $6-6-1932$ & Salyer & $\mathrm{CA}$ & 4 & 123.583 & 40.890 \\
\hline $6-6-1932$ & Scott Bar & CA & 4 & 123.003 & 41.742 \\
\hline $6-6-1932$ & Shasta & CA & 4 & 122.491 & 40.59 \\
\hline
\end{tabular}


Table 1. Modified Mercalli Intensity Observations for California North-Coast

Earthquakes

\begin{tabular}{|c|c|c|c|c|c|}
\hline Mo-Day-Yr & Location & St & MMI & Long $\left({ }^{\circ} W\right)$ & $\operatorname{Lat}\left({ }^{\circ} \mathrm{N}\right)$ \\
\hline $6-6-1932$ & Smith River & $\mathrm{CA}$ & 4 & 124.146 & 41.928 \\
\hline $6-6-1932$ & Trinity Alps & $\mathrm{CA}$ & 4 & 122.889 & 40.858 \\
\hline $6-6-1932$ & Trinity Center & $\mathrm{CA}$ & 4 & 122.700 & 41.010 \\
\hline $6-6-1932$ & Ukiah & $\mathrm{CA}$ & 4 & 123.207 & 39.150 \\
\hline $6-6-1932$ & Waddington & $\mathrm{CA}$ & 4 & 124.201 & 40.567 \\
\hline $6-6-1932$ & Weaverville & $\mathrm{CA}$ & 4 & 122.941 & 40.731 \\
\hline $6-6-1932$ & Weed & $\mathrm{CA}$ & 4 & 122.385 & 41.423 \\
\hline $6-6-1932$ & Whitlow & $\mathrm{CA}$ & 4 & 123.796 & 40.315 \\
\hline $6-6-1932$ & Willits & $\mathrm{CA}$ & 4 & 123.354 & 39.410 \\
\hline $6-6-1932$ & Yreka & $\mathrm{CA}$ & 4 & 122.633 & 41.736 \\
\hline $6-6-1932$ & Zenia & $\mathrm{CA}$ & 4 & 123.491 & 40.206 \\
\hline $6-6-1932$ & Bodega & $\mathrm{CA}$ & 3 & 122.973 & 38.345 \\
\hline $6-6-1932$ & Branscomb & $\mathrm{CA}$ & 3 & 123.624 & 39.654 \\
\hline $6-6-1932$ & Callahan & $\mathrm{CA}$ & 3 & 122.800 & 41.310 \\
\hline $6-6-1932$ & Calpella & $\mathrm{CA}$ & 3 & 123.203 & 39.234 \\
\hline $6-6-1932$ & Challenge & $\mathrm{CA}$ & 3 & 121.223 & 39.488 \\
\hline $6-6-1932$ & Dorris & $\mathrm{CA}$ & 3 & 121.917 & 41.968 \\
\hline $6-6-1932$ & Fern & $\mathrm{CA}$ & 3 & 121.931 & 40.688 \\
\hline $6-6-1932$ & Forest & $\mathrm{CA}$ & 3 & 120.852 & 39.491 \\
\hline $6-6-1932$ & Forest Glen & $\mathrm{CA}$ & 3 & 123.324 & 40.373 \\
\hline $6-6-1932$ & Foresthill & $\mathrm{CA}$ & 3 & 120.817 & 39.020 \\
\hline $6-6-1932$ & Gerber & $\mathrm{CA}$ & 3 & 122.149 & 40.056 \\
\hline $6-6 \cdot 1932$ & Grenada & $\mathrm{CA}$ & 3 & 122.519 & 41.647 \\
\hline $6-6-1932$ & Hamburg & $\mathrm{CA}$ & 3 & 123.059 & 41.783 \\
\hline $6-6-1932$ & Hayfork & $C A$ & 3 & 123.182 & 40.554 \\
\hline $6-6-1932$ & Hazel Creek & $\mathrm{CA}$ & 3 & 122.360 & 41.070 \\
\hline $6-6-1932$ & Hilt & $\mathrm{CA}$ & 3 & 122.622 & 41.995 \\
\hline $6-6-1932$ & Hornbrook & $\mathrm{CA}$ & 3 & 122.555 & 41.910 \\
\hline $6-6-1932$ & Inwood & $\mathrm{CA}$ & 3 & 121.956 & 40.524 \\
\hline $6-6-1932$ & Larkspur & $\mathrm{CA}$ & 3 & 122.512 & 37.938 \\
\hline $6-6-1932$ & Laytonville & $\mathrm{CA}$ & 3 & 123.482 & 39.688 \\
\hline $6-6-1932$ & Lewiston & $\mathrm{CA}$ & 3 & 122.806 & 40.708 \\
\hline $6-6-1932$ & Log Cabin & $\mathrm{CA}$ & 3 & 121.070 & 39.440 \\
\hline $6-6-1932$ & Magalia & $\mathrm{CA}$ & 3 & 121.577 & 39.812 \\
\hline $6-6-1932$ & Old Station & $\mathrm{CA}$ & 3 & 121.430 & 40.675 \\
\hline $6-6-1932$ & Quincy & $\mathrm{CA}$ & 3 & 120.946 & 39.937 \\
\hline $6-6-1932$ & Red Bluff & $\mathrm{CA}$ & 3 & 122.235 & 40.179 \\
\hline $6-6-1932$ & San Francisco & $\mathrm{CA}$ & 3 & 122.418 & 37.775 \\
\hline $6-6-1932$ & Sattley & $\mathrm{CA}$ & 3 & 120.426 & 39.616 \\
\hline $6-6-1932$ & Seiad Valley & $\mathrm{CA}$ & 3 & 123.191 & 41.841 \\
\hline $6-6-1932$ & Tomales & $\mathrm{CA}$ & 3 & 122.904 & 38.246 \\
\hline $6-6-1932$ & Twain & $\mathrm{CA}$ & 3 & 121.071 & 40.020 \\
\hline $6-6-1932$ & White Horse & $\mathrm{CA}$ & 3 & 121.397 & 41.311 \\
\hline $6-6-1932$ & Yuba City & $\mathrm{CA}$ & 3 & 121.616 & 39.141 \\
\hline $7-6-1934$ & Crescent City & $\mathrm{CA}$ & 5 & 124.201 & 41.756 \\
\hline $7-6-1934$ & Eureka & CA & 5 & 124.163 & 40.802 \\
\hline
\end{tabular}




\begin{tabular}{|c|c|c|c|c|c|}
\hline Mo-Day-Yr & Location & St & MMI & Long $\left({ }^{\circ} W\right)$ & $\operatorname{Lat}\left({ }^{\circ} \mathbf{N}\right)$ \\
\hline $7-6-1934$ & Arcata & CA & 4 & 124.082 & 40.867 \\
\hline $7-6-1934$ & Bridgeville & $\mathrm{CA}$ & 4 & 123.799 & 40.469 \\
\hline $7-6-1934$ & Carlotta & CA & 4 & 124.059 & 40.538 \\
\hline $7-6-1934$ & Fortuna & CA & 4 & 124.156 & 40.598 \\
\hline $7-6-1934$ & Gold Beach & CR & 4 & 124.421 & 42.408 \\
\hline $7-6-1934$ & North Bend & OR & 4 & 124.223 & 43.407 \\
\hline $7-6-1934$ & Petrolia & $\mathrm{CA}$ & 4 & 124.286 & 40.326 \\
\hline $7-6-1934$ & Scotia & CA & 4 & 124.100 & 40.483 \\
\hline $7-6-1934$ & Benbow & CA & 3 & 123.783 & 40.069 \\
\hline $7-6-1934$ & Brookings & QR & 3 & 124.283 & 42.053 \\
\hline $7-6-1934$ & Cape Mendocino & $C A$ & 3 & 124.406 & 40.440 \\
\hline $7-6-1934$ & Charleston & CR & 3 & 124.329 & 43.340 \\
\hline $7-6-1934$ & Grants Pass & CR & 3 & 123.327 & 42.439 \\
\hline $7-6-1934$ & Kneeland & $\mathrm{CA}$ & 3 & 123.994 & 40.761 \\
\hline $7-6-1934$ & Orick & $\mathrm{CA}$ & 3 & 124.059 & 41.287 \\
\hline $6-3-1936$ & Ferndale & CA & 5 & 124.263 & 40.576 \\
\hline $6-3-1936$ & Punta Gorda Light Station & $\mathrm{CA}$ & 5 & 124.349 & 40.249 \\
\hline $6-3-1936$ & Upper Mattole & CA & 5 & 124.165 & 40.252 \\
\hline $6-3-1936$ & Weott & $\mathrm{CA}$ & 5 & 123.921 & 40.322 \\
\hline $6-3-1936$ & Arcata & CA & 4 & 124.082 & 40.867 \\
\hline $6-3-1936$ & Bayside & $\mathrm{CA}$ & 4 & 124.063 & 40.843 \\
\hline $6-3-1936$ & Benbow & CA & 4 & 123.783 & 40.069 \\
\hline $6-3-1936$ & Blocksburg & CA & 4 & 123.635 & 40.276 \\
\hline $6-3-1936$ & Briceland & CA & 4 & 123.899 & 40.108 \\
\hline $6-3-1936$ & Bridgeville & CA & 4 & 123.799 & 40.469 \\
\hline $6-3-1936$ & Cape Mendocino & CA & 4 & 124.406 & 40.440 \\
\hline $6-3-1936$ & Capetown & CA & 4 & 124.366 & 40.466 \\
\hline $6-3-1936$ & Eureka & CA & 4 & 124.163 & 40.802 \\
\hline $6-3-1936$ & Fernbridge & CA & 4 & 124.200 & 40.616 \\
\hline $6-3-1936$ & Fields Landing & $C A$ & 4 & 124.214 & 40.725 \\
\hline $6-3-1936$ & Fort Bragg & CA & 4 & 123.804 & 39.446 \\
\hline $6-3-1936$ & Fortuna & CA & 4 & 124.156 & 40.598 \\
\hline $6-3-1936$ & Garberville & CA & 4 & 123.794 & 40.100 \\
\hline $6-3-1936$ & Harris & CA & 4 & 123.658 & 40.084 \\
\hline $6-3-1936$ & Holmes & $C A$ & 4 & 123.939 & 40.419 \\
\hline $6-3-1936$ & Korbel & CA & 4 & 123.957 & 40.871 \\
\hline $6-3-1936$ & Philo & CA & 4 & 123.444 & 39.066 \\
\hline $6-3-1936$ & Rohnerville & CA & 4 & 124.134 & 40.567 \\
\hline $6-3-1936$ & Samoa & CA & 4 & 124.185 & 40.819 \\
\hline $6-3-1936$ & Scotia & CA & 4 & 124.100 & 40.483 \\
\hline $6-3-1936$ & Trinidad & CA & 4 & 124.142 & 41.059 \\
\hline 6-3-1936 & Westport & CA & 4 & 123.782 & 39.636 \\
\hline $6-3-1936$ & Weymouth Inn & CA & 4 & 124.165 & 40.531 \\
\hline $6-3-1936$ & Whitlow & CA & 4 & 123.796 & 40.315 \\
\hline $6-3-1936$ & Alderpoint & $\mathrm{CA}$ & 3 & 123.611 & 40.176 \\
\hline $6-3-1936$ & Alton & $C A$ & 3 & 124.140 & 40.548 \\
\hline
\end{tabular}




\begin{tabular}{|c|c|c|c|c|c|}
\hline Mo-Day-Yr & Location & St & MMI & Long $\left({ }^{\circ} \mathrm{W}\right)$ & $\operatorname{Lat}\left({ }^{\circ} \mathbf{N}\right)$ \\
\hline $6-3-1936$ & Branscomb & $\mathrm{CA}$ & 3 & 123.624 & 39.654 \\
\hline $6-3-1936$ & Carlotta & $\mathrm{CA}$ & 3 & 124.059 & 40.538 \\
\hline $6-3-1936$ & Comptche & $\mathrm{CA}$ & 3 & 123.590 & 39.265 \\
\hline $6-3-1936$ & Crescent City & $\mathrm{CA}$ & 3 & 124.201 & 41.756 \\
\hline $6-3-1936$ & Dos Rios & $\mathrm{CA}$ & 3 & 123.352 & 39.717 \\
\hline $6-3-1936$ & Ettersburg & $\mathrm{CA}$ & 3 & 123.996 & 40.139 \\
\hline $6-3-1936$ & Hyampom & $\mathrm{CA}$ & 3 & 123.451 & 40.618 \\
\hline $6-3-1936$ & Hydesville & $\mathrm{CA}$ & 3 & 124.096 & 40.548 \\
\hline $6-3-1936$ & Little River & $\mathrm{CA}$ & 3 & 123.787 & 39.271 \\
\hline $6-3-1936$ & Manchester & $\mathrm{CA}$ & 3 & 123.687 & 38.970 \\
\hline $6-3-1936$ & Petrolia & CA & 3 & 124.286 & 40.326 \\
\hline $6-3-1936$ & Piercy & $\mathrm{CA}$ & 3 & 123.794 & 39.966 \\
\hline $6-3-1936$ & Redwood Valley & $\mathrm{CA}$ & 3 & 123.203 & 39.266 \\
\hline $6-3-1936$ & Rio Del & $\mathrm{CA}$ & 3 & 124.105 & 40.499 \\
\hline $6-3-1936$ & Salyer & $\mathrm{CA}$ & 3 & 123.583 & 40.890 \\
\hline $6-3-1936$ & Smith River & $\mathrm{CA}$ & 3 & 124.146 & 41.928 \\
\hline $6-3-1936$ & Waddington & $\mathrm{CA}$ & 3 & 124.201 & 40.567 \\
\hline 6-3-1936 & Willets & CA & 3 & 123.354 & 39.410 \\
\hline $6-3-1936$ & Willow Creek & $\mathrm{CA}$ & 3 & 123.630 & 40.940 \\
\hline $2-9-1941$ & Arcata & CA & 6 & 124.082 & 40.867 \\
\hline 2-9-1941 & Eureka & $\mathrm{CA}$ & 6 & 124.163 & 40.802 \\
\hline $2-9-1941$ & Ferndale & CA & 6 & 124.263 & 40.576 \\
\hline 2-9-1941 & Punta Gorda Light Station & $\mathrm{CA}$ & 6 & 124.349 & 40.249 \\
\hline $2-9-1941$ & Shelter Cove & CA & 6 & 124.072 & 40.031 \\
\hline $2-9-1941$ & Bridgeville & $\mathrm{CA}$ & 5 & 123.799 & 40.469 \\
\hline $2-9-1941$ & Carlotta & $\mathrm{CA}$ & 5 & 124.059 & 40.538 \\
\hline $2-9-1941$ & Crescent City & $\mathrm{CA}$ & 5 & 124.201 & 41.756 \\
\hline 2-9-1941 & Fields Landing & CA & 5 & 124.214 & 40.725 \\
\hline 2-9-1941 & Forest Glen & CA & 5 & 123.324 & 40.373 \\
\hline $2-9-1941$ & Fort Seward & $\mathrm{CA}$ & 5 & 123.642 & 40.223 \\
\hline 2-9-1941 & Fortuna & $\mathrm{CA}$ & 5 & 124.156 & 40.598 \\
\hline 2-9-1941 & Garberville & $\mathrm{CA}$ & 5 & 123.794 & 40.100 \\
\hline 2-9-1941 & Klamath & CA & 5 & 124.037 & 41.527 \\
\hline 2-9-1941 & Loleta & CA & 5 & 124.224 & 40.641 \\
\hline 2-9-1941 & Orick & CA & 5 & 124.059 & 41.287 \\
\hline $2-9-1941$ & Orleans & CA & 5 & 123.540 & 41.302 \\
\hline $2-9-1941$ & Scotia & $\mathrm{CA}$ & 5 & 124.100 & 40.483 \\
\hline 2-9-1941 & Trinidad & CA & 5 & 124.142 & 41.059 \\
\hline $2-9-1941$ & Upper Mattole & CA & 5 & 124.165 & 40.252 \\
\hline $2-9-1941$ & Weott & $\mathrm{CA}$ & 5 & 123.921 & 40.322 \\
\hline $2-9-1941$ & Willow Creek & $\mathrm{CA}$ & 5 & 123.630 & 40.940 \\
\hline 2-9-1941 & Alderpoint & $\mathrm{CA}$ & 4 & 123.611 & 40.176 \\
\hline $2-9-1941$ & Benbow & CA & 4 & 123.783 & 40.069 \\
\hline $2-9-1941$ & Blue Lake & $\mathrm{CA}$ & 4 & 123.983 & 40.883 \\
\hline $2-9-1941$ & Briceland & $\mathrm{CA}$ & 4 & 123.899 & 40.108 \\
\hline $2-9-1941$ & Brookings & QR & 4 & 124.283 & 42.053 \\
\hline
\end{tabular}


Earthquakes

\begin{tabular}{|c|c|c|c|c|c|}
\hline Mo-Day-Yr & Location & St & MMI & Long $\left({ }^{\circ} W\right)$ & Lat $\left({ }^{\circ} \mathbf{N}\right)$ \\
\hline 2-9-1941 & Cape Mendocino & CA & 4 & 124.406 & 40.440 \\
\hline $2-9-1941$ & Ettersburg & CA & 4 & 123.996 & 40.139 \\
\hline $2-9-1941$ & Glendale & CR & 4 & 123.422 & 42.736 \\
\hline $2-9-1941$ & Harris & CA & 4 & 123.658 & 40.084 \\
\hline $2-9-1941$ & Honeydew & $\mathrm{CA}$ & 4 & 124.122 & 40.244 \\
\hline $2-9-1941$ & Hoopa & $\mathrm{CA}$ & 4 & 123.673 & 41.051 \\
\hline $2-9-1941$ & Hyampom & CA & 4 & 123.451 & 40.618 \\
\hline $2-9-1941$ & Island Mountain & CA & 4 & 123.489 & 40.026 \\
\hline 2-9-1941 & Mendocino & CA & 4 & 123.798 & 39.308 \\
\hline $2-9-1941$ & Miranda & $\mathrm{CA}$ & 4 & 123.823 & 40.235 \\
\hline $2-9-1941$ & Mount Shasta & CA & 4 & 122.309 & 41.310 \\
\hline $2-9-1941$ & O'Brien & CR & 4 & 123.702 & 42.068 \\
\hline $2-9-1941$ & Point Arena & CA & 4 & 123.692 & 38.909 \\
\hline $2-9-1941$ & Rockport & CA & 4 & 123.815 & 39.739 \\
\hline $2-9-1941$ & Smith River & $\mathrm{CA}$ & 4 & 124.146 & 41.928 \\
\hline $2-9-1941$ & Yager & CA & 4 & 123.820 & 40.540 \\
\hline $2-9-1941$ & Zenia & $\mathrm{CA}$ & 4 & 123.491 & 40.206 \\
\hline $2-9-1941$ & Blocksburg & CA & 3 & 123.635 & 40.276 \\
\hline 2-9-1941 & Cummings & CA & 3 & 123.631 & 39.833 \\
\hline $2-9-1941$ & Fort Bragg & $\mathrm{CA}$ & 3 & 123.804 & 39.446 \\
\hline $2-9-1941$ & Gold Beach & OR & 3 & 124.421 & 42.408 \\
\hline $2-9-1941$ & Laytonville & CA & 3 & 123.482 & 39.688 \\
\hline 2-9-1941 & Longvale & CA & 3 & 123.429 & 39.555 \\
\hline $2-9-1941$ & Sawyers Bar & CA & 3 & 123.130 & 41.300 \\
\hline $2-9-1941$ & Weaverville & $\mathrm{CA}$ & 3 & 122.941 & 40.731 \\
\hline $2-9-1941$ & Willets & CA & 3 & 123.354 & 39.410 \\
\hline 2-9-1941 & Yreka & $\mathrm{CA}$ & 3 & 122.633 & 41.736 \\
\hline 10-3-1941 & Eureka & CA & 7 & 124.163 & 40.802 \\
\hline $10-3-1941$ & Ferndale & CA & 6 & 124.263 & 40.576 \\
\hline 10-3-1941 & Fields Landing & CA & 6 & 124.214 & 40.725 \\
\hline $10-3-1941$ & Korbel & $\mathrm{CA}$ & 6 & 123.957 & 40.871 \\
\hline $10-3-1941$ & Pepperwood & CA & 6 & 123.992 & 40.446 \\
\hline $10-3-1941$ & Rio Dell & $\mathrm{CA}$ & 6 & 124.105 & 40.499 \\
\hline $10-3-1941$ & Rockport & CA & 6 & 123.815 & 39.739 \\
\hline $10-3-1941$ & Upper Mattole & $\mathrm{CA}$ & 6 & 124.165 & 40.252 \\
\hline $10-3-1941$ & Arcata & $\mathrm{CA}$ & 5 & 124.082 & 40.867 \\
\hline 10-3-1941 & Benbow & $\mathrm{CA}$ & 5 & 123.783 & 40.069 \\
\hline $10-3-1941$ & Briceland & CA & 5 & 123.899 & 40.108 \\
\hline $10-3-1941$ & Cape Mendocino & CA & 5 & 124.406 & 40.440 \\
\hline $10-3-1941$ & Carlotta & CA & 5 & 124.059 & 40.538 \\
\hline $10-3-1941$ & Dyerville & $\mathrm{CA}$ & 5 & 123.925 & 40.356 \\
\hline $10-3-1941$ & Garberville & CA & 5 & 123.794 & 40.100 \\
\hline $10-3-1941$ & Holmes & $\mathrm{CA}$ & 5 & 123.939 & 40.419 \\
\hline $10-3-1941$ & Hyampom & $\mathrm{CA}$ & 5 & 123.451 & 40.618 \\
\hline $10-3-1941$ & Kneeland & $\mathrm{CA}$ & 5 & 123.994 & 40.761 \\
\hline $10-3-1941$ & Loleta & $\mathrm{CA}$ & 5 & 124.224 & 40.64 \\
\hline
\end{tabular}


Table 1. Modified Mercalli Intensity Observations for California North-Coast Earthquakes

\begin{tabular}{|c|c|c|c|c|c|}
\hline Mo-Day-Yr & Location & St & MMI & Long $\left({ }^{\circ} W\right)$ & Lat $\left({ }^{\circ} N\right)$ \\
\hline $10-3-1941$ & Petrolia & $\mathrm{CA}$ & 5 & 124.286 & 40.326 \\
\hline 10-3-1941 & Wildwood & $\mathrm{CA}$ & 5 & 123.053 & 40.400 \\
\hline $10-3-1941$ & Alderpoint & $\mathrm{CA}$ & 4 & 123.611 & 40.176 \\
\hline $10-3-1941$ & Blue Lake & $\mathrm{CA}$ & 4 & 123.983 & 40.883 \\
\hline $10-3-1941$ & Branscomb & $\mathrm{CA}$ & 4 & 123.624 & 39.654 \\
\hline $10-3-1941$ & Bridgeville & CA & 4 & 123.799 & 40.469 \\
\hline $10-3-1941$ & Burnt Ranch & $\mathrm{CA}$ & 4 & 123.473 & 40.809 \\
\hline $10-3-1941$ & Capetown & $\mathrm{CA}$ & 4 & 124.366 & 40.466 \\
\hline $10-3-1941$ & Cummings & $\mathrm{CA}$ & 4 & 123.631 & 39.833 \\
\hline $10-3-1941$ & Denny & $\mathrm{CA}$ & 4 & 123.386 & 40.944 \\
\hline 10-3-1941 & Dunsmuir & $\mathrm{CA}$ & 4 & 122.271 & 41.208 \\
\hline $10-3-1941$ & Elk & $\mathrm{CA}$ & 4 & 123.717 & 39.130 \\
\hline $10-3-1941$ & Ettersburg & $\mathrm{CA}$ & 4 & 123.996 & 40.139 \\
\hline 10-3-1941 & Forest Glen & $\mathrm{CA}$ & 4 & 123.324 & 40.373 \\
\hline 10-3-1941 & Fort Bragg & CA & 4 & 123.804 & 39.446 \\
\hline $10-3-1941$ & Fortuna & $\mathrm{CA}$ & 4 & 124.156 & 40.598 \\
\hline $10-3-1941$ & Happy Camp & $\mathrm{CA}$ & 4 & 123.378 & 41.793 \\
\hline $10-3-1941$ & Harris & $\mathrm{CA}$ & 4 & 123.658 & 40.084 \\
\hline 10-3-1941 & Hartsook & $\mathrm{CA}$ & 4 & 123.790 & 40.010 \\
\hline 10-3-1941 & Hayfork & $\mathrm{CA}$ & 4 & 123.182 & 40.554 \\
\hline $10-3-1941$ & Honeydew & $\mathrm{CA}$ & 4 & 124.122 & 40.244 \\
\hline $10-3-1941$ & Hoopa & $\mathrm{CA}$ & 4 & 123.673 & 41.051 \\
\hline 10-3-1941 & Island Mountain & $\mathrm{CA}$ & 4 & 123.489 & 40.026 \\
\hline $10-3-1941$ & Klamath & CA & 4 & 124.037 & 41.527 \\
\hline $10-3-1941$ & Little River & CA & 4 & 123.787 & 39.271 \\
\hline 10-3-1941 & Longvale & $\mathrm{CA}$ & 4 & 123.429 & 39.555 \\
\hline 10-3-1941 & Miranda & CA & 4 & 123.823 & 40.235 \\
\hline $10-3-1941$ & Mount Shasta & $\mathrm{CA}$ & 4 & 122.309 & 41.310 \\
\hline $10-3-1941$ & Orick & $\mathrm{CA}$ & 4 & 124.059 & 41.287 \\
\hline $10-3-1941$ & Orland & $\mathrm{CA}$ & 4 & 122.195 & 39.748 \\
\hline $10-3-1941$ & Orleans & $\mathrm{CA}$ & 4 & 123.540 & 41.302 \\
\hline $10-3-1941$ & Piercy & $\mathrm{CA}$ & 4 & 123.794 & 39.966 \\
\hline $10-3-1941$ & Point Arena & $\mathrm{CA}$ & 4 & 123.692 & 38.909 \\
\hline $10-3-1941$ & Salyer & $\mathrm{CA}$ & 4 & 123.583 & 40.890 \\
\hline $10-3-1941$ & San Francisco & $\mathrm{CA}$ & 4 & 122.418 & 37.775 \\
\hline $10-3-1941$ & Sawyers Bar & $\mathrm{CA}$ & 4 & 123.130 & 41.300 \\
\hline 10-3-1941 & Scotia & $\mathrm{CA}$ & 4 & 124.100 & 40.483 \\
\hline 10-3-1941 & Smith River & $\mathrm{CA}$ & 4 & 124.146 & 41.928 \\
\hline $10-3-1941$ & Somes Bar & $\mathrm{CA}$ & 4 & 123.475 & 41.376 \\
\hline $10-3-1941$ & Trinidad & $\mathrm{CA}$ & 4 & 124.142 & 41.059 \\
\hline $10-3-1941$ & Ukiah & $\mathrm{CA}$ & 4 & 123.207 & 39.150 \\
\hline $10-3-1941$ & Weaverville & $\mathrm{CA}$ & 4 & 122.941 & 40.731 \\
\hline $10-3-1941$ & Weitchpec & $\mathrm{CA}$ & 4 & 123.707 & 41.188 \\
\hline 10-3-1941 & Weott & $\mathrm{CA}$ & 4 & 123.921 & 40.322 \\
\hline $10-3-1941$ & Willits & $\mathrm{CA}$ & 4 & 123.354 & 39.410 \\
\hline $10-3-1941$ & Willow Creek & CA & 4 & 123.630 & 40.940 \\
\hline
\end{tabular}


Earthquakes

\begin{tabular}{|c|c|c|c|c|c|}
\hline Mo-Day-Yr & Location & St & MMI & Long $\left({ }^{\circ} \mathrm{W}\right)$ & Lat $\left({ }^{\circ} \mathbf{N}\right)$ \\
\hline 10-3-1941 & Willows & CA & 4 & 122.193 & 39.524 \\
\hline $10-3-1941$ & Blocksburg & $\mathrm{CA}$ & 3 & 123.635 & 40.276 \\
\hline $10-3-1941$ & Crescent City & $\mathrm{CA}$ & 3 & 124.201 & 41.756 \\
\hline $10-3-1941$ & Fort Jones & $\mathrm{CA}$ & 3 & 122.839 & 41.608 \\
\hline $10-3-1941$ & Junction City & CA & 3 & 123.053 & 40.733 \\
\hline $10-3-1941$ & Mad River & $\mathrm{CA}$ & 3 & 123.500 & 40.450 \\
\hline $10-3-1941$ & San Rafael & $\mathrm{CA}$ & 3 & 122.530 & 37.974 \\
\hline $10-3-1941$ & Spyrock & $\mathrm{CA}$ & 3 & 123.443 & 39.877 \\
\hline $10-3-1941$ & Stinson Beach & CA & 3 & 122.643 & 37.901 \\
\hline $10-3-1941$ & Vina & $\mathrm{CA}$ & 3 & 122.053 & 39.933 \\
\hline $10-3-1941$ & Zenia & $\mathrm{CA}$ & 3 & 123.491 & 40.206 \\
\hline $5-19-1945$ & Upper Mattole & $\mathrm{CA}$ & 5 & 124.165 & 40.252 \\
\hline 5-19-1945 & Arcata & $\mathrm{CA}$ & 4 & 124.082 & 40.867 \\
\hline $5-19-1945$ & Blue Lake & $\mathrm{CA}$ & 4 & 123.983 & 40.883 \\
\hline 5-19-1945 & Carlotta & $\mathrm{CA}$ & 4 & 124.059 & 40.538 \\
\hline 5-19-1945 & Eureka & $\mathrm{CA}$ & 4 & 124.163 & 40.802 \\
\hline 5-19-1945 & Ferndale & $\mathrm{CA}$ & 4 & 124.263 & 40.576 \\
\hline 5-19-1945 & Fields Landing & $\mathrm{CA}$ & 4 & 124.214 & 40.725 \\
\hline 5-19-1945 & Klamath & $\mathrm{CA}$ & 4 & 124.037 & 41.527 \\
\hline 5-19-1945 & Orick & $\mathrm{CA}$ & 4 & 124.059 & 41.287 \\
\hline 5-19-1945 & Piercy & $\mathrm{CA}$ & 4 & 123.794 & 39.966 \\
\hline $5-19-1945$ & Scotia & $\mathrm{CA}$ & 4 & 124.100 & 40.483 \\
\hline $5-19-1945$ & Briceland & $\mathrm{CA}$ & 3 & 123.899 & 40.108 \\
\hline $5-19-1945$ & Fort Bragg & $\mathrm{CA}$ & 3 & 123.804 & 39.446 \\
\hline 5-19-1945 & Kneeland & $\mathrm{CA}$ & 3 & 123.994 & 40.761 \\
\hline 5-19-1945 & Smith River & $\mathrm{CA}$ & 3 & 124.146 & 41.928 \\
\hline 5-19-1945 & Willits & $\mathrm{CA}$ & 3 & 123.354 & 39.410 \\
\hline $10-8-1951$ & Alton & $\mathrm{CA}$ & 7 & 124.140 & 40.548 \\
\hline 10-8-1951 & Bridgeville & $\mathrm{CA}$ & 7 & 123.799 & 40.469 \\
\hline $10-8-1951$ & Grizzly Bluff & $\mathrm{CA}$ & 7 & 124.170 & 40.562 \\
\hline $10-8-1951$ & Rio Dell & $\mathrm{CA}$ & 7 & 124.105 & 40.499 \\
\hline $10-8-1951$ & Scotia & $\mathrm{CA}$ & 7 & 124.100 & 40.483 \\
\hline $10-8-1951$ & Weott & $\mathrm{CA}$ & 7 & 123.921 & 40.322 \\
\hline $10-8-1951$ & Alderpoint & $\mathrm{CA}$ & 6 & 123.611 & 40.176 \\
\hline $10-8-1951$ & Benbow & $\mathrm{CA}$ & 6 & 123.783 & 40.069 \\
\hline $10-8-1951$ & Blocksburg & $\mathrm{CA}$ & 6 & 123.635 & 40.276 \\
\hline $10-8-1951$ & Carlotta & $\mathrm{CA}$ & 6 & 124.059 & 40.538 \\
\hline $10-8-1951$ & Ettersburg & $\mathrm{CA}$ & 6 & 123.996 & 40.139 \\
\hline $10-8-1951$ & Fortuna & $\mathrm{CA}$ & 6 & 124.156 & 40.598 \\
\hline $10-8-1951$ & Garberville & $\mathrm{CA}$ & 6 & 123.794 & 40.100 \\
\hline $10-8-1951$ & Petrolia & $\mathrm{CA}$ & 6 & 124.286 & 40.326 \\
\hline $10-8-1951$ & Upper Mattole & $\mathrm{CA}$ & 6 & 124.165 & 40.252 \\
\hline $10-8-1951$ & Arcata & $\mathrm{CA}$ & 5 & 124.082 & 40.867 \\
\hline $10-8-1951$ & Blue Lake & $\mathrm{CA}$ & 5 & 123.983 & 40.883 \\
\hline $10-8-1951$ & Corning & $\mathrm{CA}$ & 5 & 122.178 & 39.928 \\
\hline $10-8-1951$ & Covelo & $\mathrm{CA}$ & 5 & 123.247 & 39.793 \\
\hline
\end{tabular}


Table 1. Modified Mercalli Intensity Observations for California North-Coast

\section{Earthquakes}

\begin{tabular}{|c|c|c|c|c|c|}
\hline Mo-Day-Yr & Location & St & MMI & Long $\left({ }^{\circ} W\right)$ & $\operatorname{Lat}\left({ }^{\circ} \mathbf{N}\right)$ \\
\hline $10-8-1951$ & Eureka & $\mathrm{CA}$ & 5 & 124.163 & 40.802 \\
\hline $10-8-1951$ & Ferndale & CA & 5 & 124.263 & 40.576 \\
\hline 10-8-1951 & Fields Landing & $\mathrm{CA}$ & 5 & 124.214 & 40.725 \\
\hline $10-8-1951$ & Hayfork & CA & 5 & 123.182 & 40.554 \\
\hline $10-8-1951$ & Hyampom & $\mathrm{CA}$ & 5 & 123.451 & 40.618 \\
\hline $10-8-1951$ & Laytonville & $\mathrm{CA}$ & 5 & 123.482 & 39.688 \\
\hline 10-8-1951 & Miranda & $\mathrm{CA}$ & 5 & 123.823 & 40.235 \\
\hline 10-8-1951 & Piercy & $\mathrm{CA}$ & 5 & 123.794 & 39.966 \\
\hline $10-8-1951$ & Red Bluff & CA & 5 & 122.235 & 40.179 \\
\hline $10-8-1951$ & Westport & $\mathrm{CA}$ & 5 & 123.782 & 39.636 \\
\hline $10-8-1951$ & Willits & $\mathrm{CA}$ & 5 & 123.354 & 39.410 \\
\hline $10-8-1951$ & Willows & $\mathrm{CA}$ & 5 & 122.193 & 39.524 \\
\hline $10-8-1951$ & Big Bar & $\mathrm{CA}$ & 4 & 123.255 & 40.741 \\
\hline 10-8-1951 & Burnt Ranch & $\mathrm{CA}$ & 4 & 123.473 & 40.809 \\
\hline $10-8-1951$ & Comptche & $\mathrm{CA}$ & 4 & 123.590 & 39.265 \\
\hline $10-8-1951$ & Cummings & CA & 4 & 123.631 & 39.833 \\
\hline $10-8-1951$ & Denny & $\mathrm{CA}$ & 4 & 123.386 & 40.944 \\
\hline $10-8-1951$ & Elk & $\mathrm{CA}$ & 4 & 123.717 & 39.130 \\
\hline $10-8-1951$ & Flournoy & $\mathrm{CA}$ & 4 & 122.435 & 39.921 \\
\hline $10-8-1951$ & Fort Bragg & $\mathrm{CA}$ & 4 & 123.804 & 39.446 \\
\hline $10-8-1951$ & Glenn & $\mathrm{CA}$ & 4 & 122.013 & 39.522 \\
\hline 10-8-1951 & Kneeland & $\mathrm{CA}$ & 4 & 123.994 & 40.761 \\
\hline $10-8-1951$ & Lake Mountain & CA & 4 & 123.390 & 40.130 \\
\hline $10-8-1951$ & Orick & $\mathrm{CA}$ & 4 & 124.059 & 41.287 \\
\hline $10-8-1951$ & Orland & CA & 4 & 122.195 & 39.748 \\
\hline $10-8-1951$ & Richvale & $\mathrm{CA}$ & 4 & 121.744 & 39.494 \\
\hline 10-8-1951 & Rockport & $\mathrm{CA}$ & 4 & 123.815 & 39.739 \\
\hline 10-8-1951 & Ruth & $\mathrm{CA}$ & 4 & 123.320 & 40.270 \\
\hline $10-8-1951$ & Spyrock & $\mathrm{CA}$ & 4 & 123.443 & 39.877 \\
\hline $10-8-1951$ & Trinidad & CA & 4 & 124.142 & 41.059 \\
\hline $10-8-1951$ & Vina & CA & 4 & 122.053 & 39.933 \\
\hline $10-8-1951$ & Willow Creek & CA & 4 & 123.630 & 40.940 \\
\hline $10-8-1951$ & Manchester & $\mathrm{CA}$ & 3 & 123.687 & 38.970 \\
\hline $10-8-1951$ & Paskenta & $\mathrm{CA}$ & 3 & 122.545 & 39.885 \\
\hline $10-8-1951$ & Tehama & $\mathrm{CA}$ & 3 & 122.122 & 40.027 \\
\hline $11-25-1954$ & Bridgeville & CA & 5 & 123.799 & 40.469 \\
\hline $11-25-1954$ & Burnt Ranch & $\mathrm{CA}$ & 5 & 123.473 & 40.809 \\
\hline $11-25-1954$ & Carlotta & CA & 5 & 124.059 & 40.538 \\
\hline $11-25-1954$ & Eureka & $\mathrm{CA}$ & 5 & 124.163 & 40.802 \\
\hline $11-25-1954$ & Ferndale & $\mathrm{CA}$ & 5 & 124.263 & 40.576 \\
\hline $11-25-1954$ & Fields Landing & $\mathrm{CA}$ & 5 & 124.214 & 40.725 \\
\hline $11-25-1954$ & Hoopa & CA & 5 & 123.673 & 41.051 \\
\hline $11-25-1954$ & Hyampom & $\mathrm{CA}$ & 5 & 123.451 & 40.618 \\
\hline $11-25-1954$ & Korbel & $\mathrm{CA}$ & 5 & 123.957 & 40.871 \\
\hline $11-25-1954$ & Loleta & $\mathrm{CA}$ & 5 & 124.224 & 40.641 \\
\hline $11-25-1954$ & Orick & CA & 5 & 124.059 & 41.287 \\
\hline
\end{tabular}


Table 1. Modified Mercalli Intensity Observations for California North-Coast Earthquakes

\begin{tabular}{|c|c|c|c|c|c|}
\hline Mo-Day-Yr & Location & St & MMI & Long $\left({ }^{\circ} W\right)$ & $\operatorname{Lat}\left({ }^{\circ} \mathbf{N}\right)$ \\
\hline $11-25-1954$ & Pepperwood & $\mathrm{CA}$ & 5 & 123.992 & 40.446 \\
\hline $11-25-1954$ & Scotia & $\mathrm{CA}$ & 5 & 124.100 & 40.483 \\
\hline $11-25-1954$ & Arcata & $\mathrm{CA}$ & 4 & 124.082 & 40.867 \\
\hline $11-25-1954$ & Fort Bragg & $\mathrm{CA}$ & 4 & 123.804 & 39.446 \\
\hline $11-25-1954$ & Fortuna & $\mathrm{CA}$ & 4 & 124.156 & 40.598 \\
\hline $11-25-1954$ & Garberville & $\mathrm{CA}$ & 4 & 123.794 & 40.100 \\
\hline $11-25-1954$ & Holmes & $\mathrm{CA}$ & 4 & 123.939 & 40.419 \\
\hline $11-25-1954$ & Miranda & $\mathrm{CA}$ & 4 & 123.823 & 40.235 \\
\hline $11-25-1954$ & Orleans & $\mathrm{CA}$ & 4 & 123.540 & 41.302 \\
\hline $11-25-1954$ & Piercy & $\mathrm{CA}$ & 4 & 123.794 & 39.966 \\
\hline $11-25-1954$ & Point Arena & $\mathrm{CA}$ & 4 & 123.692 & 38.909 \\
\hline $11-25-1954$ & Rockport & $\mathrm{CA}$ & 4 & 123.815 & 39.739 \\
\hline $11-25-1954$ & Samoa & $\mathrm{CA}$ & 4 & 124.185 & 40.819 \\
\hline $11-25-1954$ & Weott & $\mathrm{CA}$ & 4 & 123.921 & 40.322 \\
\hline $11-25-1954$ & Willow Creek & $\mathrm{CA}$ & 4 & 123.630 & 40.940 \\
\hline $11-25-1954$ & Junction City & $\mathrm{CA}$ & 3 & 123.053 & 40.733 \\
\hline $11-25-1954$ & San Francisco & $\mathrm{CA}$ & 3 & 122.418 & 37.775 \\
\hline $11-25-1954$ & Spyrock & $\mathrm{CA}$ & 3 & 123.443 & 39.877 \\
\hline $11-25-1954$ & Trinidad & $\mathrm{CA}$ & 3 & 124.142 & 41.059 \\
\hline $11-25-1954$ & Willits & $\mathrm{CA}$ & 3 & 123.354 & 39.410 \\
\hline $12-21-1954$ & Arcata & CA & 7 & 124.082 & 40.867 \\
\hline $12-21-1954$ & Bayside & $\mathrm{CA}$ & 7 & 124.063 & 40.843 \\
\hline $12-21-1954$ & Blue Lake & $\mathrm{CA}$ & 7 & 123.983 & 40.883 \\
\hline $12-21-1954$ & Brainard & $\mathrm{CA}$ & 7 & 124.109 & 40.812 \\
\hline $12-21-1954$ & Crannell & $\mathrm{CA}$ & 7 & 124.084 & 41.012 \\
\hline $12-21-1954$ & Eureka & $\mathrm{CA}$ & 7 & 124.163 & 40.802 \\
\hline $12-21-1954$ & Ferndale & $\mathrm{CA}$ & 7 & 124.263 & 40.576 \\
\hline $12-21-1954$ & Fieldbrook & CA & 7 & 124.034 & 40.966 \\
\hline $12-21-1954$ & Fields Landing & CA & 7 & 124.214 & 40.725 \\
\hline $12-21-1954$ & Fortuna & CA & 7 & 124.156 & 40.598 \\
\hline $12-21-1954$ & Korbel & $\mathrm{CA}$ & 7 & 123.957 & 40.871 \\
\hline $12-21-1954$ & Mckinleyville & $\mathrm{CA}$ & 7 & 124.099 & 40.947 \\
\hline $12-21-1954$ & Rio Dell & $\mathrm{CA}$ & 7 & 124.105 & 40.499 \\
\hline $12-21-1954$ & Rohnerville & $\mathrm{CA}$ & 7 & 124.134 & 40.567 \\
\hline $12-21-1954$ & Samoa & CA & 7 & 124.185 & 40.819 \\
\hline $12-21-1954$ & Alton & $\mathrm{CA}$ & 6 & 124.140 & 40.548 \\
\hline $12-21-1954$ & Blocksburg & $\mathrm{CA}$ & 6 & 123.635 & 40.276 \\
\hline $12-21-1954$ & Briceland & $\mathrm{CA}$ & 6 & 123.899 & 40.108 \\
\hline $12-21-1954$ & Bridgeville & $\mathrm{CA}$ & 6 & 123.799 & 40.469 \\
\hline $12-21-1954$ & Brookings & OR & 6 & 124.283 & 42.053 \\
\hline $12-21-1954$ & Burnt Ranch & CA & 6 & 123.473 & 40.809 \\
\hline $12-21-1954$ & Carlotta & $\mathrm{CA}$ & 6 & 124.059 & 40.538 \\
\hline $12-21-1954$ & Castella & CA & 6 & 122.317 & 41.139 \\
\hline $12-21-1954$ & Denny & $\mathrm{CA}$ & 6 & 123.386 & 40.944 \\
\hline $12-21-1954$ & Dows Prairie & $\mathrm{CA}$ & 6 & 124.100 & 40.974 \\
\hline $12-21-1954$ & Dyerville & CA & 6 & 123.925 & 40.356 \\
\hline
\end{tabular}


Table 1. Modified Mercalli Intensity Observations for California North-Coast

Earthquakes

\begin{tabular}{|c|c|c|c|c|c|}
\hline Mo-Day-Yr & Location & St & MMI & Long $\left({ }^{\circ} W\right)$ & $\operatorname{Lat}\left({ }^{\circ} \mathbf{N}\right)$ \\
\hline $12-21-1954$ & Fernbridge & $\mathrm{CA}$ & 6 & 124.200 & 40.616 \\
\hline 12-21-1954 & Fort Seward & $\mathrm{CA}$ & 6 & 123.642 & 40.223 \\
\hline $12-21-1954$ & Freshwater & $\mathrm{CA}$ & 6 & 124.061 & 40.762 \\
\hline $12-21-1954$ & Gerber & $\mathrm{CA}$ & 6 & 122.149 & 40.056 \\
\hline $12-21-1954$ & Hayfork & $\mathrm{CA}$ & 6 & 123.182 & 40.554 \\
\hline $12-21-1954$ & Holmes & $\mathrm{CA}$ & 6 & 123.939 & 40.419 \\
\hline $12-21-1954$ & Hoopa & CA & 6 & 123.673 & 41.051 \\
\hline $12-21-1954$ & Hyampom & CA & 6 & 123.451 & 40.618 \\
\hline $12-21-1954$ & Hydesville & $\mathrm{CA}$ & 6 & 124.096 & 40.548 \\
\hline $12-21-1954$ & Kekawaka & $\mathrm{CA}$ & 6 & 123.518 & 40.096 \\
\hline $12-21-1954$ & Klamath & $\mathrm{CA}$ & 6 & 124.037 & 41.527 \\
\hline $12-21-1954$ & Loleta & $\mathrm{CA}$ & 6 & 124.224 & 40.641 \\
\hline $12-21-1954$ & Mccloud & CA & 6 & 122.138 & 41.256 \\
\hline $12-21-1954$ & Miranda & CA & 6 & 123.823 & 40.235 \\
\hline $12-21-1954$ & Orick & $\mathrm{CA}$ & 6 & 124.059 & 41.287 \\
\hline $12-21-1954$ & Orland & $\mathrm{CA}$ & 6 & 122.195 & 39.748 \\
\hline $12-21-1954$ & Orleans & $\mathrm{CA}$ & 6 & 123.540 & 41.302 \\
\hline $12-21-1954$ & Pepperwood & $\mathrm{CA}$ & 6 & 123.992 & 40.446 \\
\hline $12-21-1954$ & Piercy & $\mathrm{CA}$ & 6 & 123.794 & 39.966 \\
\hline $12-21-1954$ & Proberta & $\mathrm{CA}$ & 6 & 122.169 & 40.082 \\
\hline $12-21-1954$ & Red Bluff & $\mathrm{CA}$ & 6 & 122.235 & 40.179 \\
\hline $12-21-1954$ & Redding & $\mathrm{CA}$ & 6 & 122.391 & 40.587 \\
\hline $12-21-1954$ & Sawyers Bar & $\mathrm{CA}$ & 6 & 123.130 & 41.300 \\
\hline $12-21-1954$ & Scotia & $\mathrm{CA}$ & 6 & 124.100 & 40.483 \\
\hline $12-21-1954$ & Showers Pass & CA & 6 & 123.755 & 40.604 \\
\hline $12-21-1954$ & Trinidad & $\mathrm{CA}$ & 6 & 124.142 & 41.059 \\
\hline $12-21-1954$ & Waddington & $\mathrm{CA}$ & 6 & 124.201 & 40.567 \\
\hline $12-21-1954$ & Weaverville & $\mathrm{CA}$ & 6 & 122.941 & 40.731 \\
\hline $12-21-1954$ & Weott & $\mathrm{CA}$ & 6 & 123.921 & 40.322 \\
\hline $12-21-1954$ & Willits & $\mathrm{CA}$ & 6 & 123.354 & 39.410 \\
\hline $12-21-1954$ & Willow Creek & $\mathrm{CA}$ & 6 & 123.630 & 40.940 \\
\hline $12-21-1954$ & Alderpoint & $\mathrm{CA}$ & 5 & 123.611 & 40.176 \\
\hline $12-21-1954$ & Butte Valley & $\mathrm{CA}$ & 5 & 121.933 & 41.949 \\
\hline $12-21-1954$ & Cecilville & $\mathrm{CA}$ & 5 & 123.139 & 41.141 \\
\hline $12-21-1954$ & Corning & $\mathrm{CA}$ & 5 & 122.178 & 39.928 \\
\hline $12-21-1954$ & Covelo & $\mathrm{CA}$ & 5 & 123.247 & 39.793 \\
\hline $12-21-1954$ & Crescent City & $\mathrm{CA}$ & 5 & 124.201 & 41.756 \\
\hline $12-21-1954$ & Cummings & $\mathrm{CA}$ & 5 & 123.631 & 39.833 \\
\hline $12-21-1954$ & Eel Rock & $\mathrm{CA}$ & 5 & 123.730 & 40.290 \\
\hline $12-21-1954$ & Ettersburg & $\mathrm{CA}$ & 5 & 123.996 & 40.139 \\
\hline $12-21-1954$ & Fort Jones & $\mathrm{CA}$ & 5 & 122.839 & 41.608 \\
\hline 12-21-1954 & Garberville & $\mathrm{CA}$ & 5 & 123.794 & 40.100 \\
\hline $12-21-1954$ & Junction City & CA & 5 & 123.053 & 40.733 \\
\hline $12-21-1954$ & Klamath Falls & OR & 5 & 121.781 & 42.225 \\
\hline 12-21-1954 & Lake Mountain & $\mathrm{CA}$ & 5 & 123.390 & 40.130 \\
\hline $12-21-1954$ & Laytonville & $\mathrm{CA}$ & 5 & 123.482 & 39.688 \\
\hline
\end{tabular}


Table 1. Modified Mercalli Intensity Observations for California North-Coast

Earthquakes

\begin{tabular}{|c|c|c|c|c|c|}
\hline Mo-Day-Yr & Location & St & MMI & Long $\left({ }^{\circ} \mathrm{W}\right)$ & $\operatorname{Lat}\left({ }^{\circ} \mathrm{N}\right)$ \\
\hline $12-21-1954$ & Macdoel & CA & 5 & 122.004 & 41.827 \\
\hline $12-21-1954$ & Merrill & OR & 5 & 121.599 & 42.025 \\
\hline $12-21-1954$ & Mineral & CA & 5 & 121.594 & 40.348 \\
\hline $12-21-1954$ & Nubieber & CA & 5 & 121.182 & 41.096 \\
\hline $12-21-1954$ & Phillipsville & $\mathrm{CA}$ & 5 & 123.785 & 40.209 \\
\hline $12-21-1954$ & Requa & CA & 5 & 124.065 & 41.547 \\
\hline $12-21-1954$ & Richardson Grove & $\mathrm{CA}$ & 5 & 123.790 & 40.020 \\
\hline $12-21-1954$ & Rockport & $\mathrm{CA}$ & 5 & 123.815 & 39.739 \\
\hline $12-21-1954$ & Round Mountain & CA & 5 & 121.941 & 40.794 \\
\hline $12-21-1954$ & Scott Bar & $\mathrm{CA}$ & 5 & 123.003 & 41.742 \\
\hline $12-21-1954$ & Tehama & CA & 5 & 122.122 & 40.027 \\
\hline $12-21-1954$ & Trinity Center & $\mathrm{CA}$ & 5 & 122.700 & 41.010 \\
\hline $12-21-1954$ & Tulelake & $\mathrm{CA}$ & 5 & 121.476 & 41.956 \\
\hline $12-21-1954$ & Weed & $\mathrm{CA}$ & 5 & 122.385 & 41.423 \\
\hline $12-21-1954$ & Woodland & $\mathrm{CA}$ & 5 & 121.772 & 38.679 \\
\hline $12-21-1954$ & Yreka & $\mathrm{CA}$ & 5 & 122.633 & 41.736 \\
\hline $12-21-1954$ & Auburn & $\mathrm{CA}$ & 4 & 121.076 & 38.897 \\
\hline $12-21-1954$ & Bieber & CA & 4 & 121.143 & 41.121 \\
\hline $12-21-1954$ & Brooks & $\mathrm{CA}$ & 4 & 122.147 & 38.742 \\
\hline $12-21-1954$ & Capay & $\mathrm{CA}$ & 4 & 122.047 & 38.708 \\
\hline $12-21-1954$ & Cave Junction & CR & 4 & 123.647 & 42.163 \\
\hline $12-21-1954$ & Colusa & $\mathrm{CA}$ & 4 & 122.008 & 39.214 \\
\hline $12-21-1954$ & Coos Bay & CR & 4 & 124.216 & 43.368 \\
\hline $12-21-1954$ & Davis Creek & $\mathrm{CA}$ & 4 & 120.371 & 41.733 \\
\hline $12-21-1954$ & Etna & CA & 4 & 122.894 & 41.457 \\
\hline $12-21-1954$ & Gazelle & $\mathrm{CA}$ & 4 & 122.519 & 41.521 \\
\hline $12-21-1954$ & Gold Beach & CR & 4 & 124.421 & 42.408 \\
\hline $12-21-1954$ & Grenada & $\mathrm{CA}$ & 4 & 122.519 & 41.647 \\
\hline $12-21-1954$ & Happy Camp & $\mathrm{CA}$ & 4 & 123.378 & 41.793 \\
\hline $12-21-1954$ & Harbor & OR & 4 & 124.266 & 42.053 \\
\hline $12-21-1954$ & Island Mountain & $\mathrm{CA}$ & 4 & 123.489 & 40.026 \\
\hline $12-21-1954$ & Las Plumas & $\mathrm{CA}$ & 4 & 121.485 & 39.674 \\
\hline $12-21-1954$ & Lewiston & $\mathrm{CA}$ & 4 & 122.806 & 40.708 \\
\hline $12-21-1954$ & Los Molinos & $\mathrm{CA}$ & 4 & 122.099 & 40.021 \\
\hline $12-21-1954$ & Macarthur & $\mathrm{CA}$ & 4 & 121.398 & 41.050 \\
\hline 12-21-1954 & Manton & CA & 4 & 121.869 & 40.435 \\
\hline 12-21-1954 & Medford & CR & 4 & 122.874 & 42.327 \\
\hline $12-21-1954$ & Modoc Point & OR & 4 & 121.867 & 42.445 \\
\hline $12-21-1954$ & Mount Hebron & CA & 4 & 122.003 & 41.787 \\
\hline $12-21-1954$ & Mount Shasta & $\mathrm{CA}$ & 4 & 122.309 & 41.310 \\
\hline $12-21-1954$ & O'Brien & CR & 4 & 123.702 & 42.068 \\
\hline $12-21-1954$ & Ono & CA & 4 & 122.617 & 40.475 \\
\hline $12-21-1954$ & Pistol River & CR & 4 & 124.394 & 42.277 \\
\hline $12-21-1954$ & Platina & $C A$ & 4 & 122.890 & 40.360 \\
\hline $12-21-1954$ & Ruth & CA & 4 & 123.320 & 40.270 \\
\hline $12-21-1954$ & Selma & OR & 4 & 123.615 & 42.279 \\
\hline
\end{tabular}


Table 1. Modified Mercalli Intensity Observations for California North-Coast Earthquakes

\begin{tabular}{|c|c|c|c|c|c|}
\hline Mo-Day-Yr & Location & St & MMI & Long $\left({ }^{\circ} W\right)$ & $\operatorname{Lat}\left({ }^{\circ} \mathrm{N}\right)$ \\
\hline $12-21-1954$ & Upper Mattole & $\mathrm{CA}$ & 4 & 124.165 & 40.252 \\
\hline $12-21-1954$ & Vina & $\mathrm{CA}$ & 4 & 122.053 & 39.933 \\
\hline $12-21-1954$ & Westport & $\mathrm{CA}$ & 4 & 123.782 & 39.636 \\
\hline $12-21-1954$ & Alturas & $\mathrm{CA}$ & 3 & 120.541 & 41.487 \\
\hline $12-21-1954$ & Bonanza & OR & 3 & 121.405 & 42.199 \\
\hline $12-21-1954$ & Calpella & $\mathrm{CA}$ & 3 & 123.203 & 39.234 \\
\hline $12-21-1954$ & Chico & $\mathrm{CA}$ & 3 & 121.836 & 39.729 \\
\hline $12-21-1954$ & Clements & $\mathrm{CA}$ & 3 & 121.087 & 38.191 \\
\hline $12-21-1954$ & Coquille & CR & 3 & 124.186 & 43.177 \\
\hline $12-21-1954$ & Denmark & OR & 3 & 124.461 & 42.891 \\
\hline $12-21-1954$ & Finley & $\mathrm{CA}$ & 3 & 122.874 & 39.004 \\
\hline $12-21-1954$ & Flourney & $\mathrm{CA}$ & 3 & 120.297 & 41.235 \\
\hline $12-21-1954$ & Gridley & $\mathrm{CA}$ & 3 & 121.693 & 39.364 \\
\hline $12-21-1954$ & Hartsook & $\mathrm{CA}$ & 3 & 123.790 & 40.010 \\
\hline $12-21-1954$ & Hat Creek & $\mathrm{CA}$ & 3 & 121.500 & 40.790 \\
\hline $12-21-1954$ & Lakeport & $\mathrm{CA}$ & 3 & 122.915 & 39.043 \\
\hline $12-21-1954$ & Ophir & OR & 3 & 124.382 & 42.563 \\
\hline $12-21-1954$ & Pondosa & $\mathrm{CA}$ & 3 & 121.688 & 41.199 \\
\hline $12-21-1954$ & Project City & $\mathrm{CA}$ & 3 & 122.351 & 40.680 \\
\hline $12-21-1954$ & Richfield & $\mathrm{CA}$ & 3 & 122.176 & 39.975 \\
\hline $12-21-1954$ & San Francisco & $\mathrm{CA}$ & 3 & 122.418 & 37.775 \\
\hline $12-21-1954$ & Sitkum & OR & 3 & 123.860 & 43.148 \\
\hline $12-21-1954$ & Sprague River & OR & 3 & 121.503 & 42.456 \\
\hline $12-21-1954$ & Takilma & OR & 3 & 123.618 & 42.052 \\
\hline $12-21-1954$ & Tionesta & $\mathrm{CA}$ & 3 & 121.327 & 41.646 \\
\hline $12-21-1954$ & Ukiah & $\mathrm{CA}$ & 3 & 123.207 & 39.150 \\
\hline $12-21-1954$ & Upper Lake & $\mathrm{CA}$ & 3 & 122.909 & 39.165 \\
\hline $10-11-1956$ & Bayside & $\mathrm{CA}$ & 5 & 124.063 & 40.843 \\
\hline $10-11-1956$ & Blue Lake & $\mathrm{CA}$ & 5 & 123.983 & 40.883 \\
\hline $10-11-1956$ & Crannell & $\mathrm{CA}$ & 5 & 124.084 & 41.012 \\
\hline $10-11-1956$ & Eureka & $\mathrm{CA}$ & 5 & 124.163 & 40.802 \\
\hline $10-11-1956$ & Ferndale & $\mathrm{CA}$ & 5 & 124.263 & 40.576 \\
\hline 10-11-1956 & Fields Landing & $\mathrm{CA}$ & 5 & 124.214 & 40.725 \\
\hline $10-11-1956$ & Fortuna & $\mathrm{CA}$ & 5 & 124.156 & 40.598 \\
\hline $10-11-1956$ & Korbel & CA & 5 & 123.957 & 40.871 \\
\hline $10-11-1956$ & Loleta & $\mathrm{CA}$ & 5 & 124.224 & 40.641 \\
\hline $10-11-1956$ & Petrolia & $\mathrm{CA}$ & 5 & 124.286 & 40.326 \\
\hline $10-11-1956$ & Rio Dell & $\mathrm{CA}$ & 5 & 124.105 & 40.499 \\
\hline $10-11-1956$ & Alton & $\mathrm{CA}$ & 4 & 124.140 & 40.548 \\
\hline $10-11-1956$ & Blocksburg & $\mathrm{CA}$ & 4 & 123.635 & 40.276 \\
\hline $10-11-1956$ & Bridgeville & $\mathrm{CA}$ & 4 & 123.799 & 40.469 \\
\hline $10-11-1956$ & Denny & $\mathrm{CA}$ & 4 & 123.386 & 40.944 \\
\hline $10-11-1956$ & Forest Glen & $C A$ & 4 & 123.324 & 40.373 \\
\hline $10-11-1956$ & Fort Bragg & $C A$ & 4 & 123.804 & 39.446 \\
\hline $10-11-1956$ & Freshwater & $C A$ & 4 & 124.061 & 40.762 \\
\hline $10-11-1956$ & Garberville & $\mathrm{CA}$ & 4 & 123.794 & 40.100 \\
\hline
\end{tabular}




\begin{tabular}{|c|c|c|c|c|c|}
\hline Mo-Day-Yr & Location & St & MMI & Long $\left({ }^{\circ} \mathrm{W}\right)$ & $\operatorname{Lat}\left({ }^{\circ} \mathbf{N}\right)$ \\
\hline $10-11-1956$ & Holmes & $\mathrm{CA}$ & 4 & 123.939 & 40.419 \\
\hline 10-11-1956 & Honeydew & $\mathrm{CA}$ & 4 & 124.122 & 40.244 \\
\hline $10-11-1956$ & Kneeland & $\mathrm{CA}$ & 4 & 123.994 & 40.761 \\
\hline $10-11-1956$ & Piercy & $\mathrm{CA}$ & 4 & 123.794 & 39.966 \\
\hline 10-11-1956 & Scotia & $\mathrm{CA}$ & 4 & 124.100 & 40.483 \\
\hline 10-11-1956 & Trinidad & $\mathrm{CA}$ & 4 & 124.142 & 41.059 \\
\hline 10-11-1956 & Alderpoint & $\mathrm{CA}$ & 3 & 123.611 & 40.176 \\
\hline 10-11-1956 & Arcata & $\mathrm{CA}$ & 3 & 124.082 & 40.867 \\
\hline 10-11-1956 & Carlotta & $\mathrm{CA}$ & 3 & 124.059 & 40.538 \\
\hline 10-11-1956 & Crescent City & $\mathrm{CA}$ & 3 & 124.201 & 41.756 \\
\hline 10-11-1956 & Ettersburg & $\mathrm{CA}$ & 3 & 123.996 & 40.139 \\
\hline 10-11-1956 & Medford & OR & 3 & 122.874 & 42.327 \\
\hline 10-11-1956 & Phillipsville & $\mathrm{CA}$ & 3 & 123.785 & 40.209 \\
\hline 10-11-1956 & Weitchpec & $\mathrm{CA}$ & 3 & 123.707 & 41.188 \\
\hline $10-11-1956$ & Willow Creek & $\mathrm{CA}$ & 3 & 123.630 & 40.940 \\
\hline $8-9-1960$ & Comptche & CA & 5 & 123.590 & 39.265 \\
\hline $8-9-1960$ & Cummings & CA & 5 & 123.631 & 39.833 \\
\hline $8-9-1960$ & Ferndale & CA & 5 & 124.263 & 40.576 \\
\hline $8-9-1960$ & Fort Bragg & $\mathrm{CA}$ & 5 & 123.804 & 39.446 \\
\hline $8-9-1960$ & Fortuna & $\mathrm{CA}$ & 5 & 124.156 & 40.598 \\
\hline 8-9-1960 & Casper & CA & 4 & 123.815 & 39.364 \\
\hline $8-9-1960$ & Crannell & $\mathrm{CA}$ & 4 & 124.084 & 41.012 \\
\hline $8-9-1960$ & Elk & CA & 4 & 123.717 & 39.130 \\
\hline $8-9-1960$ & Eureka & CA & 4 & 124.163 & 40.802 \\
\hline $8-9-1960$ & Fields Landing & $\mathrm{CA}$ & 4 & 124.214 & 40.725 \\
\hline $8-9-1960$ & Honeydew & $\mathrm{CA}$ & 4 & 124.122 & 40.244 \\
\hline $8-9-1960$ & Hoopa & $C A$ & 4 & 123.673 & 41.051 \\
\hline $8-9-1960$ & Hyampom & CA & 4 & 123.451 & 40.618 \\
\hline 8-9-1960 & Korbel & $C A$ & 4 & 123.957 & 40.871 \\
\hline 8-9-1960 & Lakeport & $\mathrm{CA}$ & 4 & 122.915 & 39.043 \\
\hline $8-9-1960$ & Mendocino & CA & 4 & 123.798 & 39.308 \\
\hline $8-9-1960$ & Orick & CA & 4 & 124.059 & 41.287 \\
\hline $8-9-1960$ & Petrolia & CA & 4 & 124.286 & 40.326 \\
\hline 8-9-1960 & Rio Dell & CA & 4 & 124.105 & 40.499 \\
\hline $8-9-1960$ & San Francisco & $\mathrm{CA}$ & 4 & 122.418 & 37.775 \\
\hline 8-9-1960 & Scotia & $\mathrm{CA}$ & 4 & 124.100 & 40.483 \\
\hline $8-9-1960$ & Upper Lake & $\mathrm{CA}$ & 4 & 122.909 & 39.165 \\
\hline $8-9-1960$ & Willits & CA & 4 & 123.354 & 39.410 \\
\hline 8-23-1962 & Brookings & $\alpha R$ & 6 & 124.283 & 42.053 \\
\hline 8-23-1962 & Cave Junction & $O R$ & 6 & 123.647 & 42.163 \\
\hline 8-23-1962 & Crescent City & $\mathrm{CA}$ & 6 & 124.201 & 41.756 \\
\hline $8-23-1962$ & Smith River & CA & 6 & 124.146 & 41.928 \\
\hline 8-23-1962 & Bayside & CA & 5 & 124.063 & 40.843 \\
\hline $8-23-1962$ & Cecilville & $\mathrm{CA}$ & 5 & 123.139 & 41.141 \\
\hline 8-23-1962 & Edgewood & $\mathrm{CA}$ & 5 & 122.431 & 41.458 \\
\hline $8-23-1962$ & Ferndale & CA & 5 & 124.263 & 40.576 \\
\hline
\end{tabular}


Table 1. Modified Mercalli Intensity Observations for California North-Coast

Earthquakes

\begin{tabular}{|c|c|c|c|c|c|}
\hline Mo-Day-Yr & Location & St & MMI & Long $\left({ }^{\circ} \mathrm{W}\right)$ & $\operatorname{Lat}\left({ }^{\circ} \mathbf{N}\right)$ \\
\hline 8-23-1962 & Fields Landing & $\mathrm{CA}$ & 5 & 124.214 & 40.725 \\
\hline $8-23-1962$ & Fort Dick & $\mathrm{CA}$ & 5 & 124.148 & 41.868 \\
\hline $8-23-1962$ & Freshwater & $\mathrm{CA}$ & 5 & 124.061 & 40.762 \\
\hline 8-23-1962 & Gasquet & $\mathrm{CA}$ & 5 & 123.968 & 41.846 \\
\hline 8-23-1962 & Grants Pass & OR & 5 & 123.327 & 42.439 \\
\hline $8-23-1962$ & Happy Camp & $\mathrm{CA}$ & 5 & 123.378 & 41.793 \\
\hline 8-23-1962 & Harbor & OR & 5 & 124.266 & 42.053 \\
\hline 8-23-1962 & Holmes & CA & 5 & 123.939 & 40.419 \\
\hline 8-23-1962 & Klamath & $\mathrm{CA}$ & 5 & 124.037 & 41.527 \\
\hline 8-23-1962 & Korbel & $\mathrm{CA}$ & 5 & 123.957 & 40.871 \\
\hline $8-23-1962$ & Merlin & OR & 5 & 123.419 & 42.518 \\
\hline $8-23-1962$ & Orleans & $\mathrm{CA}$ & 5 & 123.540 & 41.302 \\
\hline 8-23-1962 & Pistol River & OR & 5 & 124.394 & 42.277 \\
\hline 8-23-1962 & Requa & $\mathrm{CA}$ & 5 & 124.065 & 41.547 \\
\hline 8-23-1962 & Salyer & CA & 5 & 123.583 & 40.890 \\
\hline 8-23-1962 & Selma & OR & 5 & 123.615 & 42.279 \\
\hline 8-23-1962 & Agness & OR & 4 & 124.068 & 42.557 \\
\hline 8-23-1962 & Arcata & $\mathrm{CA}$ & 4 & 124.082 & 40.867 \\
\hline 8-23-1962 & Bandon & OR & 4 & 124.410 & 43.120 \\
\hline $8-23-1962$ & Briceland & $\mathrm{CA}$ & 4 & 123.899 & 40.108 \\
\hline 8-23-1962 & Covelo & CA & 4 & 123.247 & 39.793 \\
\hline 8-23-1962 & Crannell & CA & 4 & 124.084 & 41.012 \\
\hline $8-23-1962$ & Cutten & CA & 4 & 124.142 & 40.770 \\
\hline 8-23-1962 & Denny & CA & 4 & 123.386 & 40.944 \\
\hline $8-23-1962$ & Eureka & $\mathrm{CA}$ & 4 & 124.163 & 40.802 \\
\hline $8-23-1962$ & Fortuna & $\mathrm{CA}$ & 4 & 124.156 & 40.598 \\
\hline $8-23-1962$ & Gazelle & CA & 4 & 122.519 & 41.521 \\
\hline $8-23-1962$ & Gold Beach & OR & 4 & 124.421 & 42.408 \\
\hline $8-23-1962$ & Gold Hill & CR & 4 & 123.049 & 42.432 \\
\hline $8-23-1962$ & Honeydew & CA & 4 & 124.122 & 40.244 \\
\hline 8-23-1962 & Hoopa & $\mathrm{CA}$ & 4 & 123.673 & 41.051 \\
\hline 8-23-1962 & Horse Creek & $\mathrm{CA}$ & 4 & 122.996 & 41.824 \\
\hline 8-23-1962 & Island Mountain & $\mathrm{CA}$ & 4 & 123.489 & 40.026 \\
\hline 8-23-1962 & Laytonville & $\mathrm{CA}$ & 4 & 123.482 & 39.688 \\
\hline $8-23-1962$ & Loleta & CA & 4 & 124.224 & 40.641 \\
\hline $8-23-1962$ & Medford & OR & 4 & 122.874 & 42.327 \\
\hline $8-23-1962$ & Montague & CA & 4 & 122.527 & 41.728 \\
\hline 8-23-1962 & Mount Shasta & CA & 4 & 122.309 & 41.310 \\
\hline $8-23-1962$ & O'Brien & OR & 4 & 123.702 & 42.068 \\
\hline $8-23-1962$ & Ophir & CR & 4 & 124.382 & 42.563 \\
\hline $8-23-1962$ & Orick & $\mathrm{CA}$ & 4 & 124.059 & 41.287 \\
\hline $8-23-1962$ & Rio Dell & CA & 4 & 124.105 & 40.499 \\
\hline 8-23-1962 & Rohnerville & $C A$ & 4 & 124.134 & 40.567 \\
\hline $8-23-1962$ & Samoa & $\mathrm{CA}$ & 4 & 124.185 & 40.819 \\
\hline 8-23-1962 & Sawyers Bar & $\mathrm{CA}$ & 4 & 123.130 & 41.300 \\
\hline 8-23-1962 & Scotia & $\mathrm{CA}$ & 4 & 124.100 & 40.483 \\
\hline
\end{tabular}




\begin{tabular}{|c|c|c|c|c|c|}
\hline Mo-Day-Yr & Location & St & MMI & Long $\left({ }^{\circ} W\right)$ & $\operatorname{Lat}\left({ }^{\circ} \mathbf{N}\right)$ \\
\hline $8-23-1962$ & Scott Bar & CA & 4 & 123.003 & 41.742 \\
\hline $8-23-1962$ & Seiad Valley & $\mathrm{CA}$ & 4 & 123.191 & 41.841 \\
\hline 8-23-1962 & Somes Bar & $\mathrm{CA}$ & 4 & 123.475 & 41.376 \\
\hline $8-23-1962$ & Takilma & OR & 4 & 123.618 & 42.052 \\
\hline 8-23-1962 & Trinidad & CA & 4 & 124.142 & 41.059 \\
\hline 8-23-1962 & Wedderburn & OR & 4 & 124.414 & 42.433 \\
\hline $8-23-1962$ & Wilderville & CR & 4 & 123.466 & 42.383 \\
\hline 8-23-1962 & Alton & CA & 3 & 124.140 & 40.548 \\
\hline 8-23-1962 & Blue Lake & $\mathrm{CA}$ & 3 & 123.983 & 40.883 \\
\hline 8-23-1962 & Burnt Ranch & CA & 3 & 123.473 & 40.809 \\
\hline 8-23-1962 & Carlotta & $\mathrm{CA}$ & 3 & 124.059 & 40.538 \\
\hline 8-23-1962 & Coos Bay & $\alpha R$ & 3 & 124.216 & 43.368 \\
\hline 8-23-1962 & Coquelle & $O R$ & 3 & 124.186 & 43.177 \\
\hline 8-23-1962 & Etna & $\mathrm{CA}$ & 3 & 122.894 & 41.457 \\
\hline $8-23-1962$ & Ettersburg & $\mathrm{CA}$ & 3 & 123.996 & 40.139 \\
\hline $8-23-1962$ & Fort Jones & $\mathrm{CA}$ & 3 & 122.839 & 41.608 \\
\hline 8-23-1962 & Glendale & $\alpha$ & 3 & 123.422 & 42.736 \\
\hline $8-23-1962$ & Greenview & CA & 3 & 122.904 & 41.551 \\
\hline $8-23-1962$ & Grenada & CA & 3 & 122.519 & 41.647 \\
\hline $8-23-1962$ & Hyampom & CA & 3 & 123.451 & 40.618 \\
\hline $8-23-1962$ & Kneeland & $\mathrm{CA}$ & 3 & 123.994 & 40.761 \\
\hline $8-23-1962$ & Myers Flat & $\mathrm{CA}$ & 3 & 123.869 & 40.266 \\
\hline 8-23-1962 & Petrolia & CA & 3 & 124.286 & 40.326 \\
\hline 8-23-1962 & Port Orford & OR & 3 & 124.496 & 42.746 \\
\hline 8-23-1962 & Sitkum & OR & 3 & 123.860 & 43.148 \\
\hline $8-23-1962$ & Sixes & OR & 3 & 124.482 & 42.820 \\
\hline $8-23-1962$ & Weaverville & $\mathrm{CA}$ & 3 & 122.941 & 40.731 \\
\hline $8-23-1962$ & Weed & CA & 3 & 122.385 & 41.423 \\
\hline 8-23-1962 & Willow Creek & CA & 3 & 123.630 & 40.940 \\
\hline 8-23-1962 & Yreka & CA & 3 & 122.633 & 41.736 \\
\hline $11-8-1980$ & Fields Landing & $\mathrm{CA}$ & 7 & 124.214 & 40.725 \\
\hline $11-8-1980$ & Arcata & $\mathrm{CA}$ & 6 & 124.082 & 40.867 \\
\hline $11-8-1980$ & Blue Lake & $\mathrm{CA}$ & 6 & 123.983 & 40.883 \\
\hline $11-8-1980$ & Brookings & QR & 6 & 124.283 & 42.053 \\
\hline $11-8-1980$ & Eureka & $\mathrm{CA}$ & 6 & 124.163 & 40.802 \\
\hline $11-8-1980$ & Ferndale & CA & 6 & 124.263 & 40.576 \\
\hline $11-8-1980$ & Fortuna & $\mathrm{CA}$ & 6 & 124.156 & 40.598 \\
\hline $11-8-1980$ & Harbor & OR & 6 & 124.266 & 42.053 \\
\hline $11-8-1980$ & Klamath & $\mathrm{CA}$ & 6 & 124.037 & 41.527 \\
\hline $11-8-1980$ & Loleta & CA & 6 & 124.224 & 40.641 \\
\hline $11-8-1980$ & Mckinleyville & $\mathrm{CA}$ & 6 & 124.099 & 40.947 \\
\hline $11-8-1980$ & Myers Flat & CA & 6 & 123.869 & 40.266 \\
\hline $11-8-1980$ & Redway & $\mathrm{CA}$ & 6 & 123.822 & 40.120 \\
\hline $11-8-1980$ & Rio Dell & CA & 6 & 124.105 & 40.499 \\
\hline $11-8-1980$ & Samoa & $\mathrm{CA}$ & 6 & 124.185 & 40.819 \\
\hline $11-8-1980$ & Trinidad & CA & 6 & 124.142 & 41.059 \\
\hline
\end{tabular}


Table 1. Modified Mercalli Intensity Observations for California North-Coast

\section{Earthquakes}

\begin{tabular}{|c|c|c|c|c|c|}
\hline Mo-Day-Yr & Location & St & MMI & Long $\left({ }^{\circ} \mathrm{W}\right)$ & Lat $\left({ }^{\circ} N\right)$ \\
\hline $11-8-1980$ & Westhaven & CA & 6 & 124.110 & 41.036 \\
\hline $11-8-1980$ & Albany & CR & 5 & 124.068 & 42.557 \\
\hline $11-8-1980$ & Alderpoint & $C A$ & 5 & 123.611 & 40.176 \\
\hline $11-8-1980$ & Bayside & $\mathrm{CA}$ & 5 & 124.063 & 40.843 \\
\hline $11-8-1980$ & Big Bar & $\mathrm{CA}$ & 5 & 123.255 & 40.741 \\
\hline $11-8-1980$ & Blocksburg & $\mathrm{CA}$ & 5 & 123.635 & 40.276 \\
\hline $11-8-1980$ & Bridgeville & $\mathrm{CA}$ & 5 & 123.799 & 40.469 \\
\hline $11-8-1980$ & Burnt Ranch & CA & 5 & 123.473 & 40.809 \\
\hline $11-8-1980$ & Carlotta & $\mathrm{CA}$ & 5 & 124.059 & 40.538 \\
\hline $11-8-1980$ & Crescent City & CA & 5 & 124.201 & 41.756 \\
\hline $11-8-1980$ & Edgewood & $\mathrm{CA}$ & 5 & 122.431 & 41.458 \\
\hline $11-8-1980$ & Etna & $\mathrm{CA}$ & 5 & 122.894 & 41.457 \\
\hline $11-8-1980$ & Finley & $\mathrm{CA}$ & 5 & 122.874 & 39.004 \\
\hline $11-8-1980$ & Fort Dick & $\mathrm{CA}$ & 5 & 124.148 & 41.868 \\
\hline $11-8-1980$ & Garberville & CA & 5 & 123.794 & 40.100 \\
\hline $11-8-1980$ & Honeydew & $\mathrm{CA}$ & 5 & 124.122 & 40.244 \\
\hline $11-8-1980$ & Hoopa & $\mathrm{CA}$ & 5 & 123.673 & 41.051 \\
\hline $11-8-1980$ & Hydesville & $\mathrm{CA}$ & 5 & 124.096 & 40.548 \\
\hline $11-8-1980$ & Kneeland & $\mathrm{CA}$ & 5 & 123.994 & 40.761 \\
\hline $11-8-1980$ & Korbel & $\mathrm{CA}$ & 5 & 123.957 & 40.871 \\
\hline $11-8-1980$ & Mccloud & $\mathrm{CA}$ & 5 & 122.138 & 41.256 \\
\hline $11-8-1980$ & Miranda & $\mathrm{CA}$ & 5 & 123.823 & 40.235 \\
\hline $11-8-1980$ & Orick & CA & 5 & 124.059 & 41.287 \\
\hline $11-8-1980$ & Scotia & $\mathrm{CA}$ & 5 & 124.100 & 40.483 \\
\hline $11-8-1980$ & Weott & $\mathrm{CA}$ & 5 & 123.921 & 40.322 \\
\hline $11-8-1980$ & Westport & CA & 5 & 123.782 & 39.636 \\
\hline $11-8-1980$ & Whitethorn & $\mathrm{CA}$ & 5 & 123.942 & 40.024 \\
\hline $11-8-1980$ & Willits & CA & 5 & 123.354 & 39.410 \\
\hline $11-8-1980$ & Willow Creek & $\mathrm{CA}$ & 5 & 123.630 & 40.940 \\
\hline $11-8-1980$ & Albion & $\mathrm{CA}$ & 4 & 123.768 & 39.224 \\
\hline $11-8-1980$ & Alta & $\mathrm{CA}$ & 4 & 120.810 & 39.207 \\
\hline $11-8-1980$ & Anderson & $\mathrm{CA}$ & 4 & 122.297 & 40.448 \\
\hline $11-8-1980$ & Annapolis & CA & 4 & 123.369 & 38.722 \\
\hline $11-8-1980$ & Ashland & CR & 4 & 122.708 & 42.195 \\
\hline $11-8-1980$ & Bangor & $\mathrm{CA}$ & 4 & 121.404 & 39.389 \\
\hline $11-8-1980$ & Baxter & $\mathrm{CA}$ & 4 & 120.780 & 39.213 \\
\hline $11-8-1980$ & Berry Creek & $\mathrm{CA}$ & 4 & 121.402 & 39.645 \\
\hline $11-8-1980$ & Bieber & CA & 4 & 121.143 & 41.121 \\
\hline $11-8-1980$ & Branscomb & $\mathrm{CA}$ & 4 & 123.624 & 39.654 \\
\hline $11-8-1980$ & Butte City & $\mathrm{CA}$ & 4 & 121.989 & 39.465 \\
\hline $11-8-1980$ & Camptonville & $\mathrm{CA}$ & 4 & 121.048 & 39.452 \\
\hline $11-8-1980$ & Cave Junction & CR & 4 & 123.647 & 42.163 \\
\hline $11-8-1980$ & Challenge & $\mathrm{CA}$ & 4 & 121.223 & 39.488 \\
\hline $11-8-1980$ & Chicago Park & $\mathrm{CA}$ & 4 & 120.960 & 39.150 \\
\hline $11-8-1980$ & Clio & CA & 4 & 120.579 & 39.743 \\
\hline $11-8-1980$ & Colfax & $\mathrm{CA}$ & 4 & 120.952 & 39.101 \\
\hline
\end{tabular}


Table 1. Modified Mercalli Intensity Observations for California North-Coast Earthquakes

\begin{tabular}{|c|c|c|c|c|c|}
\hline Mo-Day-Yr & Location & St & MMI & Long $\left({ }^{\circ} W\right)$ & Lat $\left({ }^{\circ} \mathbf{N}\right)$ \\
\hline $11-8-1980$ & Comptche & CA & 4 & 123.590 & 39.265 \\
\hline $11-8-1980$ & Cottonwood & CA & 4 & 122.280 & 40.386 \\
\hline $11-8-1980$ & Covelo & CA & 4 & 123.247 & 39.793 \\
\hline $11-8-1980$ & Dairy & OR & 4 & 121.520 & 42.235 \\
\hline $11-8-1980$ & Davis & $\mathrm{CA}$ & 4 & 121.739 & 38.545 \\
\hline $11-8-1980$ & Dorris & CA & 4 & 121.917 & 41.968 \\
\hline $11-8-1980$ & Dos Rios & CA & 4 & 123.352 & 39.717 \\
\hline $11-8-1980$ & Douglas City & $\mathrm{CA}$ & 4 & 122.944 & 40.652 \\
\hline $11-8-1980$ & Elk & $\mathrm{CA}$ & 4 & 123.717 & 39.130 \\
\hline $11-8-1980$ & Emigrant Gap & $\mathrm{CA}$ & 4 & 120.672 & 39.297 \\
\hline $11-8-1980$ & Fairfax & CA & 4 & 122.588 & 37.987 \\
\hline $11-8-1980$ & Fall River Mills & CA & 4 & 121.437 & 41.005 \\
\hline $11-8-1980$ & Fallon & NV & 4 & 118.776 & 39.474 \\
\hline $11-8-1980$ & Forbestown & $\mathrm{CA}$ & 4 & 121.266 & 39.517 \\
\hline $11-8-1980$ & Forest Glen & $\mathrm{CA}$ & 4 & 123.324 & 40.373 \\
\hline $11-8-1980$ & Forks Of Salmon & CA & 4 & 123.330 & 41.260 \\
\hline $11-8-1980$ & Fort Bragg & CA & 4 & 123.804 & 39.446 \\
\hline $11-8-1980$ & Freestone & $\mathrm{CA}$ & 4 & 122.914 & 38.373 \\
\hline $11-8-1980$ & French Gulch & CA & 4 & 122.637 & 40.701 \\
\hline 11-8-1980 & Gasquet & $\mathrm{CA}$ & 4 & 123.968 & 41.846 \\
\hline $11-8-1980$ & Gazelle & CA & 4 & 122.519 & 41.521 \\
\hline $11-8-1980$ & Gerber & CA & 4 & 122.149 & 40.056 \\
\hline $11-8-1980$ & Glenburn & $\mathrm{CA}$ & 4 & 121.489 & 41.061 \\
\hline $11-8-1980$ & Glenn & $\mathrm{CA}$ & 4 & 122.013 & 39.522 \\
\hline $11-8-1980$ & Gold Beach & OR & 4 & 124.421 & 42.408 \\
\hline $11-8-1980$ & Gold Hill & OR & 4 & 123.049 & 42.432 \\
\hline $11-8-1980$ & Grants Pass & CR & 4 & 123.327 & 42.439 \\
\hline $11-8-1980$ & Greenview & $\mathrm{CA}$ & 4 & 122.904 & 41.551 \\
\hline $11-8-1980$ & Grenada & $\mathrm{CA}$ & 4 & 122.519 & 41.647 \\
\hline $11-8-1980$ & Gualala & $\mathrm{CA}$ & 4 & 123.527 & 38.766 \\
\hline $11-8-1980$ & Hamilton City & $\mathrm{CA}$ & 4 & 122.013 & 39.743 \\
\hline $11-8-1980$ & Horse Creek & CA & 4 & 122.996 & 41.824 \\
\hline $11-8-1980$ & Hyampom & $\mathrm{CA}$ & 4 & 123.451 & 40.618 \\
\hline $11-8-1980$ & Junction City & $\mathrm{CA}$ & 4 & 123.053 & 40.733 \\
\hline $11-8-1980$ & Klamath Falls & QR & 4 & 121.781 & 42.225 \\
\hline $11-8-1980$ & Klamath River & $\mathrm{CA}$ & 4 & 122.830 & 41.860 \\
\hline $11-8-1980$ & Lakehead & $\mathrm{CA}$ & 4 & 122.378 & 40.905 \\
\hline $11-8-1980$ & Lakeside & OR & 4 & 124.174 & 43.576 \\
\hline $11-8-1980$ & Lee Vining & $\mathrm{CA}$ & 4 & 119.121 & 37.958 \\
\hline $11-8-1980$ & Leggett & $\mathrm{CA}$ & 4 & 123.713 & 39.866 \\
\hline $11-8-1980$ & Loma Mar & $\mathrm{CA}$ & 4 & 122.308 & 37.271 \\
\hline $11-8-1980$ & Macdoel & CA & 4 & 122.004 & 41.827 \\
\hline $11-8-1980$ & Mad River & $\mathrm{CA}$ & 4 & 123.500 & 40.450 \\
\hline $11-8-1980$ & Maxwell & $\mathrm{CA}$ & 4 & 122.190 & 39.276 \\
\hline $11-8-1980$ & Medford & OR & 4 & 122.874 & 42.327 \\
\hline $11-8-1980$ & Mendocino & $\mathrm{CA}$ & 4 & 123.798 & 39.308 \\
\hline
\end{tabular}


Earthquakes

\begin{tabular}{|c|c|c|c|c|c|}
\hline Mo-Day-Yr & Location & St & MMI & Long $\left({ }^{\circ} \mathrm{W}\right)$ & $\operatorname{Lat}\left({ }^{\circ} N\right)$ \\
\hline $11-8-1980$ & Meridian & $\mathrm{CA}$ & 4 & 121.913 & 39.143 \\
\hline $11-8-1980$ & Merlin & OR & 4 & 123.419 & 42.518 \\
\hline $11-8-1980$ & Merrill & OR & 4 & 121.599 & 42.025 \\
\hline $11-8-1980$ & Minden & NV & 4 & 119.765 & 38.954 \\
\hline $11-8-1980$ & Montague & CA & 4 & 122.527 & 41.728 \\
\hline $11-8-1980$ & Montgomery Creek & $\mathrm{CA}$ & 4 & 121.923 & 40.842 \\
\hline $11-8-1980$ & Mount Shasta & $\mathrm{CA}$ & 4 & 122.309 & 41.310 \\
\hline $11-8-1980$ & Nelson & $\mathrm{CA}$ & 4 & 121.764 & 39.552 \\
\hline $11-8-1980$ & Nevada City & $\mathrm{CA}$ & 4 & 121.015 & 39.262 \\
\hline $11-8-1980$ & North San Juan & CA & 4 & 121.103 & 39.369 \\
\hline $11-8-1980$ & Norway & OR & 4 & 124.156 & 43.101 \\
\hline $11-8-1980$ & Nubieber & $\mathrm{CA}$ & 4 & 121.182 & 41.096 \\
\hline $11-8-1980$ & O'Brien & OR & 4 & 123.702 & 42.068 \\
\hline $11-8-1980$ & Oak Run & $\mathrm{CA}$ & 4 & 122.024 & 40.685 \\
\hline $11-8-1980$ & Olema & $\mathrm{CA}$ & 4 & 122.787 & 38.041 \\
\hline $11-8-1980$ & Orleans & $\mathrm{CA}$ & 4 & 123.540 & 41.302 \\
\hline $11-8-1980$ & Pacific House & CA & 4 & 120.500 & 38.770 \\
\hline $11-8-1980$ & Paisley & OR & 4 & 120.545 & 42.694 \\
\hline $11-8-1980$ & Palo Cedro & $\mathrm{CA}$ & 4 & 122.238 & 40.564 \\
\hline $11-8-1980$ & Paradise & $\mathrm{CA}$ & 4 & 121.621 & 39.760 \\
\hline $11-8-1980$ & Penngrove & $\mathrm{CA}$ & 4 & 122.666 & 38.300 \\
\hline $11-8-1980$ & Pescadero & $\mathrm{CA}$ & 4 & 122.380 & 37.255 \\
\hline $11-8-1980$ & Phillipsville & $\mathrm{CA}$ & 4 & 123.785 & 40.209 \\
\hline $11-8-1980$ & Phoenix & OR & 4 & 122.817 & 42.276 \\
\hline $11-8-1980$ & Point Arena & $\mathrm{CA}$ & 4 & 123.692 & 38.909 \\
\hline $11-8-1980$ & Powers & OR & 4 & 124.072 & 42.884 \\
\hline $11-8-1980$ & Proberta & $\mathrm{CA}$ & 4 & 122.169 & 40.082 \\
\hline $11-8-1980$ & Red Bluff & $\mathrm{CA}$ & 4 & 122.235 & 40.179 \\
\hline $11-8-1980$ & Redding & $\mathrm{CA}$ & 4 & 122.391 & 40.587 \\
\hline $11-8-1980$ & Richvale & $\mathrm{CA}$ & 4 & 121.744 & 39.494 \\
\hline $11-8-1980$ & Ruth & $\mathrm{CA}$ & 4 & 123.320 & 40.270 \\
\hline $11-8-1980$ & Salyer & $\mathrm{CA}$ & 4 & 123.583 & 40.890 \\
\hline $11-8-1980$ & San Francisco & $\mathrm{CA}$ & 4 & 122.418 & 37.775 \\
\hline $11-8-1980$ & Sawyers Bar & $\mathrm{CA}$ & 4 & 123.130 & 41.300 \\
\hline $11-8-1980$ & Scott Bar & $\mathrm{CA}$ & 4 & 123.003 & 41.742 \\
\hline $11-8-1980$ & Seiad Valley & $\mathrm{CA}$ & 4 & 123.191 & 41.841 \\
\hline $11-8-1980$ & Selma & CR & 4 & 123.615 & 42.279 \\
\hline $11-8-1980$ & Smith River & $\mathrm{CA}$ & 4 & 124.146 & 41.928 \\
\hline $11-8-1980$ & Summit City & CA & 4 & 122.401 & 40.686 \\
\hline $11-8-1980$ & Talent & OR & 4 & 122.788 & 42.246 \\
\hline $11-8-1980$ & Tehama & $\mathrm{CA}$ & 4 & 122.122 & 40.027 \\
\hline $11-8-1980$ & Tulelake & $\mathrm{CA}$ & 4 & 121.476 & 41.956 \\
\hline $11-8-1980$ & Ukiah & $\mathrm{CA}$ & 4 & 123.207 & 39.150 \\
\hline $11-8-1980$ & Vina & $\mathrm{CA}$ & 4 & 122.053 & 39.933 \\
\hline $11-8-1980$ & Wedderburn & OR & 4 & 124.414 & 42.433 \\
\hline $11-8-1980$ & Weed & $\mathrm{CA}$ & 4 & 122.385 & 41.423 \\
\hline
\end{tabular}


Table 1. Modified Mercalli Intensity Observations for California North-Coast Earthquakes

\begin{tabular}{|c|c|c|c|c|c|}
\hline Mo-Day-Yr & Location & St & MMI & Long $\left({ }^{\circ} W\right)$ & $\operatorname{Lat}\left({ }^{\circ} \mathrm{N}\right)$ \\
\hline $11-8-1980$ & Whitmore & $\mathrm{CA}$ & 4 & 121.916 & 40.629 \\
\hline $11-8-1980$ & Wildwood & $\mathrm{CA}$ & 4 & 123.053 & 40.400 \\
\hline $11-8-1980$ & Yreka & $\mathrm{CA}$ & 4 & 122.633 & 41.736 \\
\hline $11-8-1980$ & Zenia & $\mathrm{CA}$ & 4 & 123.491 & 40.206 \\
\hline $11-8-1980$ & Applegate & OR & 3 & 120.991 & 39.001 \\
\hline $11-8-1980$ & Beale Air Force Base & CA & 3 & 121.440 & 39.140 \\
\hline $11-8-1980$ & Brownsville & $\mathrm{CA}$ & 3 & 121.268 & 39.473 \\
\hline $11-8-1980$ & Castella & $\mathrm{CA}$ & 3 & 122.317 & 41.139 \\
\hline $11-8-1980$ & Cazadero & CA & 3 & 123.084 & 38.533 \\
\hline $11-8-1980$ & Chiloquin & OR & 3 & 121.865 & 42.578 \\
\hline $11-8-1980$ & Chowchilla & CA & 3 & 120.259 & 37.123 \\
\hline $11-8-1980$ & Dillon Beach & CA & 3 & 122.964 & 38.251 \\
\hline $11-8-1980$ & El Verano & $\mathrm{CA}$ & 3 & 122.491 & 38.298 \\
\hline $11-8-1980$ & Forest Knolls & CA & 3 & 122.688 & 38.015 \\
\hline $11-8-1980$ & Gold Run & $\mathrm{CA}$ & 3 & 120.855 & 39.181 \\
\hline $11-8-1980$ & Hat Creek & CA & 3 & 121.500 & 40.790 \\
\hline $11-8-1980$ & Igo & $\mathrm{CA}$ & 3 & 122.541 & 40.506 \\
\hline $11-8-1980$ & Jamestown & $\mathrm{CA}$ & 3 & 120.422 & 37.953 \\
\hline $11-8-1980$ & La Pine & OR & 3 & 121.503 & 43.671 \\
\hline $11-8-1980$ & Langlois & CR & 3 & 124.450 & 42.924 \\
\hline $11-8-1980$ & Los Molinos & $\mathrm{CA}$ & 3 & 122.099 & 40.021 \\
\hline $11-8-1980$ & Manchester & $\mathrm{CA}$ & 3 & 123.687 & 38.970 \\
\hline $11-8-1980$ & Marysville & $\mathrm{CA}$ & 3 & 121.590 & 39.146 \\
\hline $11-8-1980$ & Myrtle Creek & OR & 3 & 123.292 & 43.020 \\
\hline $11-8-1980$ & Palo Alto & CA & 3 & 122.142 & 37.442 \\
\hline $11-8-1980$ & Potter Valley & $\mathrm{CA}$ & 3 & 123.112 & 39.322 \\
\hline $11-8-1980$ & Princeton & $\mathrm{CA}$ & 3 & 122.009 & 39.403 \\
\hline $11-8-1980$ & Project City & CA & 3 & 122.351 & 40.680 \\
\hline $11-8-1980$ & Prospect & OR & 3 & 122.488 & 42.751 \\
\hline $11-8-1980$ & Rancho Cordova & $\mathrm{CA}$ & 3 & 121.302 & 38.589 \\
\hline $11-8-1980$ & Round Mountain & $\mathrm{CA}$ & 3 & 121.941 & 40.794 \\
\hline $11-8-1980$ & San Gregorio & $\mathrm{CA}$ & 3 & 122.386 & 37.327 \\
\hline $11-8-1980$ & Sea Ranch & $\mathrm{CA}$ & 3 & 123.430 & 38.680 \\
\hline $11-8-1980$ & Shasta & $\mathrm{CA}$ & 3 & 122.491 & 40.599 \\
\hline $11-8-1980$ & Sixes & OR & 3 & 124.482 & 42.820 \\
\hline $11-8-1980$ & Summer Lake & OR & 3 & 120.776 & 42.973 \\
\hline $11-8-1980$ & Sutherlin & OR & 3 & 123.311 & 43.393 \\
\hline $11-8-1980$ & Tobin & $\mathrm{CA}$ & 3 & 121.308 & 39.938 \\
\hline $11-8-1980$ & Vineburg & $\mathrm{CA}$ & 3 & 122.438 & 38.273 \\
\hline $11-8-1980$ & Whiskeytown & $\mathrm{CA}$ & 3 & 122.559 & 40.639 \\
\hline $11 \cdot 8-1980$ & Wilderville & OR & 3 & 123.466 & 42.383 \\
\hline $11-8-1980$ & Willows & $\mathrm{CA}$ & 3 & 122.193 & 39.524 \\
\hline $11-21-1986$ & Petrolia & $\mathrm{CA}$ & 7 & 124.286 & 40.326 \\
\hline $11-21-1986$ & Carlotta & $\mathrm{CA}$ & 6 & 124.059 & 40.538 \\
\hline $11-21-1986$ & Ferndale & $\mathrm{CA}$ & 6 & 124.263 & 40.576 \\
\hline $11-21-1986$ & Fortuna & $\mathrm{CA}$ & 6 & 124.156 & 40.598 \\
\hline
\end{tabular}


Table 1. Modified Mercalli Intensity Observations for California North-Coast Earthquakes

\begin{tabular}{|c|c|c|c|c|c|}
\hline Mo-Day-Yr & Location & St & MMI & Long $\left({ }^{\circ} \mathrm{W}\right)$ & $\operatorname{Lat}\left({ }^{\circ} \mathrm{N}\right)$ \\
\hline $11-21-1986$ & Honeydew & $\mathrm{CA}$ & 6 & 124.122 & 40.244 \\
\hline $11-21-1986$ & Hydesville & $\mathrm{CA}$ & 6 & 124.096 & 40.548 \\
\hline $11-21-1986$ & Scotia & $\mathrm{CA}$ & 6 & 124.100 & 40.483 \\
\hline $11-21-1986$ & Alderpoint & $\mathrm{CA}$ & 5 & 123.611 & 40.176 \\
\hline $11-21-1986$ & Arcata & $\mathrm{CA}$ & 5 & 124.082 & 40.867 \\
\hline $11-21-1986$ & Blocksburg & $\mathrm{CA}$ & 5 & 123.635 & 40.276 \\
\hline $11-21-1986$ & Cutten & $\mathrm{CA}$ & 5 & 124.142 & 40.770 \\
\hline $11-21-1986$ & Fields Landing & $\mathrm{CA}$ & 5 & 124.214 & 40.725 \\
\hline $11-21-1986$ & Loleta & $\mathrm{CA}$ & 5 & 124.224 & 40.641 \\
\hline $11-21-1986$ & Miranda & $\mathrm{CA}$ & 5 & 123.823 & 40.235 \\
\hline $11-21-1986$ & Redcrest & $\mathrm{CA}$ & 5 & 123.949 & 40.401 \\
\hline $11-21-1986$ & Redway & CA & 5 & 123.822 & 40.120 \\
\hline $11-21-1986$ & Weott & $\mathrm{CA}$ & 5 & 123.921 & 40.322 \\
\hline $11-21-1986$ & Bayside & $\mathrm{CA}$ & 4 & 124.063 & 40.843 \\
\hline $11-21-1986$ & Blue Lake & $C A$ & 4 & 123.983 & 40.883 \\
\hline $11-21-1986$ & Covelo & $\mathrm{CA}$ & 4 & 123.247 & 39.793 \\
\hline $11-21-1986$ & Eureka & $\mathrm{CA}$ & 4 & 124.163 & 40.802 \\
\hline $11-21-1986$ & Fort Bragg & $\mathrm{CA}$ & 4 & 123.804 & 39.446 \\
\hline $11-21-1986$ & Garberville & CA & 4 & 123.794 & 40.100 \\
\hline $11-21-1986$ & Kneeland & CA & 4 & 123.994 & 40.761 \\
\hline $11-21-1986$ & Myers Flat & $\mathrm{CA}$ & 4 & 123.869 & 40.266 \\
\hline $11-21-1986$ & Piercy & CA & 4 & 123.794 & 39.966 \\
\hline $11-21-1986$ & Samoa & $\mathrm{CA}$ & 4 & 124.185 & 40.819 \\
\hline $11-21-1986$ & Shelter Cove & $\mathrm{CA}$ & 4 & 124.072 & 40.031 \\
\hline $11-21-1986$ & Whitethorn & CA & 4 & 123.942 & 40.024 \\
\hline $11-21-1986$ & Bridgeville & $C A$ & 3 & 123.799 & 40.469 \\
\hline $11-21-1986$ & Burnt Ranch & $C A$ & 3 & 123.473 & 40.809 \\
\hline $11-21-1986$ & Laytonville & $\mathrm{CA}$ & 3 & 123.482 & 39.688 \\
\hline $11-21-1986$ & Mad River & $\mathrm{CA}$ & 3 & 123.500 & 40.450 \\
\hline $11-21-1986$ & Mendocino & $\mathrm{CA}$ & 3 & 123.798 & 39.308 \\
\hline $11-21-1986$ & Orick & $\mathrm{CA}$ & 3 & 124.059 & 41.287 \\
\hline $11-21-1986$ & Ruth & $\mathrm{CA}$ & 3 & 123.320 & 40.270 \\
\hline $11-21-1986$ & Salyer & CA & 3 & 123.583 & 40.890 \\
\hline $11-21-1986$ & Trinidad & CA & 3 & 124.142 & 41.059 \\
\hline $11-21-1986$ & Westhaven & $\mathrm{CA}$ & 3 & 124.110 & 41.036 \\
\hline $11-21-1986$ & Westport & $C A$ & 3 & 123.782 & 39.636 \\
\hline $11-21-1986$ & Willow Creek & $\mathrm{CA}$ & 3 & 123.630 & 40.940 \\
\hline $7-31-1987$ & Carlotta & $\mathrm{CA}$ & 6 & 124.059 & 40.538 \\
\hline $7-31-1987$ & Ferndale & $C A$ & 6 & 124.263 & 40.576 \\
\hline $7-31-1987$ & Fortuna & $\mathrm{CA}$ & 6 & 124.156 & 40.598 \\
\hline $7-31-1987$ & Petrolia & $C A$ & 6 & 124.286 & 40.326 \\
\hline $7-31-1987$ & Redway & $C A$ & 6 & 123.822 & 40.120 \\
\hline 7-31-1987 & Rio Dell & $C A$ & 6 & 124.105 & 40.499 \\
\hline $7-31-1987$ & Scotia & CA & 6 & 124.100 & 40.483 \\
\hline $7-31-1987$ & Weott & $C A$ & 6 & 123.921 & 40.322 \\
\hline $7-31-1987$ & Alderpoint & $C A$ & 5 & 123.611 & 40.176 \\
\hline
\end{tabular}


Table 1. Modified Mercalli Intensity Observations for California North-Coast

Earthquakes

\begin{tabular}{|c|c|c|c|c|c|}
\hline Mo-Day-Yr & Location & St & MMl & Long $\left({ }^{\circ} W\right)$ & Lat $\left({ }^{\circ} \mathbf{N}\right)$ \\
\hline 7-31-1987 & Blocksburg & $\mathrm{CA}$ & 5 & 123.635 & 40.276 \\
\hline $7-31-1987$ & Bridgeville & $\mathrm{CA}$ & 5 & 123.799 & 40.469 \\
\hline $7-31-1987$ & Eureka & $\mathrm{CA}$ & 5 & 124.163 & 40.802 \\
\hline $7-31-1987$ & Fields Landing & $\mathrm{CA}$ & 5 & 124.214 & 40.725 \\
\hline $7-31-1987$ & Honeydew & $\mathrm{CA}$ & 5 & 124.122 & 40.244 \\
\hline $7-31-1987$ & Hydesville & $\mathrm{CA}$ & 5 & 124.096 & 40.548 \\
\hline $7-31-1987$ & Kneeland & $\mathrm{CA}$ & 5 & 123.994 & 40.761 \\
\hline $7-31-1987$ & Loleta & $\mathrm{CA}$ & 5 & 124.224 & 40.641 \\
\hline $7-31-1987$ & Miranda & $\mathrm{CA}$ & 5 & 123.823 & 40.235 \\
\hline 7-31-1987 & Piercy & CA & 5 & 123.794 & 39.966 \\
\hline $7-31-1987$ & Samoa & $\mathrm{CA}$ & 5 & 124.185 & 40.819 \\
\hline $7-31-1987$ & Shelter Cove & $\mathrm{CA}$ & 5 & 124.072 & 40.031 \\
\hline $7-31-1987$ & Westport & $\mathrm{CA}$ & 5 & 123.782 & 39.636 \\
\hline $7-31-1987$ & Whitethorn & $\mathrm{CA}$ & 5 & 123.942 & 40.024 \\
\hline $7-31-1987$ & Arcata & $\mathrm{CA}$ & 4 & 124.082 & 40.867 \\
\hline $7-31-1987$ & Blue Lake & $\mathrm{CA}$ & 4 & 123.983 & 40.883 \\
\hline $7-31-1987$ & Branscomb & $\mathrm{CA}$ & 4 & 123.624 & 39.654 \\
\hline $7-31-1987$ & Burnt Ranch & $\mathrm{CA}$ & 4 & 123.473 & 40.809 \\
\hline $7-31-1987$ & Elk & $\mathrm{CA}$ & 4 & 123.717 & 39.130 \\
\hline $7-31-1987$ & Fort Bragg & $\mathrm{CA}$ & 4 & 123.804 & 39.446 \\
\hline $7-31-1987$ & Garberville & $\mathrm{CA}$ & 4 & 123.794 & 40.100 \\
\hline $7-31-1987$ & Laytonville & $\mathrm{CA}$ & 4 & 123.482 & 39.688 \\
\hline $7-31-1987$ & Leggett & $\mathrm{CA}$ & 4 & 123.713 & 39.866 \\
\hline $7-31-1987$ & Mckinleyville & $\mathrm{CA}$ & 4 & 124.099 & 40.947 \\
\hline $7-31-1987$ & Mendocino & $\mathrm{CA}$ & 4 & 123.798 & 39.308 \\
\hline $7-31-1987$ & Phillipsville & $\mathrm{CA}$ & 4 & 123.785 & 40.209 \\
\hline $7-31-1987$ & Salyer & $\mathrm{CA}$ & 4 & 123.583 & 40.890 \\
\hline $7-31-1987$ & Westhaven & $\mathrm{CA}$ & 4 & 124.110 & 41.036 \\
\hline $7-31-1987$ & Willow Creek & $\mathrm{CA}$ & 4 & 123.630 & 40.940 \\
\hline $7-31-1987$ & Zenia & $\mathrm{CA}$ & 4 & 123.491 & 40.206 \\
\hline $7-31-1987$ & Comptche & $\mathrm{CA}$ & 3 & 123.590 & 39.265 \\
\hline $7-31-1987$ & Covelo & $\mathrm{CA}$ & 3 & 123.247 & 39.793 \\
\hline $7-31 \cdot 1987$ & Forks Of Salmon & $\mathrm{CA}$ & 3 & 123.330 & 41.260 \\
\hline $7-31-1987$ & Orleans & $\mathrm{CA}$ & 3 & 123.540 & 41.302 \\
\hline $7-31-1987$ & Rohnert Park & $\mathrm{CA}$ & 3 & 122.700 & 38.340 \\
\hline $7-31-1987$ & Ruth & $\mathrm{CA}$ & 3 & 123.320 & 40.270 \\
\hline $7-31-1987$ & Santa Rosa & $\mathrm{CA}$ & 3 & 122.713 & 38.441 \\
\hline $7-31-1987$ & Somes Bar & $\mathrm{CA}$ & 3 & 123.475 & 41.376 \\
\hline $7-31-1987$ & Willits & $\mathrm{CA}$ & 3 & 123.354 & 39.410 \\
\hline $8-17-1991$ & Klamath & $\mathrm{CA}$ & 5 & 124.037 & 41.527 \\
\hline $8-17-1991$ & Lakeside & OR & 5 & 124.174 & 43.576 \\
\hline 8-17-1991 & North Bend & CR & 5 & 124.223 & 43.407 \\
\hline $8-17-1991$ & Phillipsville & $\mathrm{CA}$ & 5 & 123.785 & 40.209 \\
\hline $8-17-1991$ & Pistol River & OR & 5 & 124.394 & 42.277 \\
\hline $8-17-1991$ & Rio Dell & $\mathrm{CA}$ & 5 & 124.105 & 40.499 \\
\hline $8-17-1991$ & Trinidad & $\mathrm{CA}$ & 5 & 124.142 & 41.059 \\
\hline
\end{tabular}


Table 1. Modified Mercalli Intensity Observations for California North-Coast Earthquakes

\begin{tabular}{|c|c|c|c|c|c|}
\hline Mo-Day-Yr & Location & St & MMI & Long $\left({ }^{\circ} \mathrm{W}\right)$ & $\operatorname{Lat}\left({ }^{\circ} \mathrm{N}\right)$ \\
\hline $8-17-1991$ & Bridgeville & $C A$ & 4 & 123.799 & 40.469 \\
\hline 8-17-1991 & Coos Bay & CR & 4 & 124.216 & 43.368 \\
\hline $8-17-1991$ & Coquille & CR & 4 & 124.186 & 43.177 \\
\hline $8-17-1991$ & Fortuna & CA & 4 & 124.156 & 40.598 \\
\hline $8-17-1991$ & Murphy & CR & 4 & 123.332 & 42.348 \\
\hline $8-17-1991$ & Myers Flat & CA & 4 & 123.869 & 40.266 \\
\hline 8-17-1991 & Reedsport & $C R$ & 4 & 124.096 & 43.703 \\
\hline 8-17-1991 & Rogue River & CR & 4 & 123.171 & 42.436 \\
\hline $8-17-1991$ & Roseburg & CR & 4 & 123.341 & 43.217 \\
\hline $8-17-1991$ & Scottsburg & OR & 4 & 123.816 & 43.654 \\
\hline $8-17-1991$ & Sixes & OR & 4 & 124.482 & 42.820 \\
\hline $8-17-1991$ & Whitethorn & CA & 4 & 123.942 & 40.024 \\
\hline $4-25-1992$ & Ferndale & CA & 8 & 124.263 & 40.576 \\
\hline 4-25-1992 & Honeydew & CA & 8 & 124.122 & 40.244 \\
\hline 4-25-1992 & Redcrest & $\mathrm{CA}$ & 8 & 123.949 & 40.401 \\
\hline 4-25-1992 & Rio Dell & $\mathrm{CA}$ & 8 & 124.105 & 40.499 \\
\hline $4-25-1992$ & Scotia & CA & 8 & 124.100 & 40.483 \\
\hline 4-25-1992 & Eureka & CA & 7 & 124.163 & 40.802 \\
\hline 4-25-1992 & Fortuna & CA & 7 & 124.156 & 40.598 \\
\hline $4-25-1992$ & Kneeland & $\mathrm{CA}$ & 7 & 123.994 & 40.761 \\
\hline 4-25-1992 & Loleta & CA & 7 & 124.224 & 40.641 \\
\hline $4-25-1992$ & Redway & CA & 7 & 123.822 & 40.120 \\
\hline $4-25-1992$ & Whitethorn & CA & 7 & 123.942 & 40.024 \\
\hline $4-25-1992$ & Alderpoint & CA & 6 & 123.611 & 40.176 \\
\hline $4-25-1992$ & Arcata & CA & 6 & 124.082 & 40.867 \\
\hline 4-25-1992 & Blue Lake & $\mathrm{CA}$ & 6 & 123.983 & 40.883 \\
\hline $4-25-1992$ & Cutten & $\mathrm{CA}$ & 6 & 124.142 & 40.770 \\
\hline 4-25-1992 & Fields Landing & CA & 6 & 124.214 & 40.725 \\
\hline 4-25-1992 & Miranda & CA & 6 & 123.823 & 40.235 \\
\hline $4-25-1992$ & Myers Flat & CA & 6 & 123.869 & 40.266 \\
\hline $4-25-1992$ & Phillipsville & $\mathrm{CA}$ & 6 & 123.785 & 40.209 \\
\hline 4-25-1992 & Samoa & $\mathrm{CA}$ & 6 & 124.185 & 40.819 \\
\hline 4-25-1992 & Weott & $\mathrm{CA}$ & 6 & 123.921 & 40.322 \\
\hline 4-25-1992 & Bridgeville & CA & 5 & 123.799 & 40.469 \\
\hline 4-25-1992 & Crescent City & CA & 5 & 124.201 & 41.756 \\
\hline 4-25-1992 & Dos Rios & $\mathrm{CA}$ & 5 & 123.352 & 39.717 \\
\hline $4-25-1992$ & Garberville & $\mathrm{CA}$ & 5 & 123.794 & 40.100 \\
\hline $4-25-1992$ & Greenview & CA & 5 & 122.904 & 41.551 \\
\hline 4-25-1992 & Klamath & $\mathrm{CA}$ & 5 & 124.037 & 41.527 \\
\hline 4-25-1992 & Laytonville & CA & 5 & 123.482 & 39.688 \\
\hline $4-25-1992$ & Mendocino & $\mathrm{CA}$ & 5 & 123.798 & 39.308 \\
\hline 4-25-1992 & Mount Shasta & $\mathrm{CA}$ & 5 & 122.309 & 41.310 \\
\hline 4-25-1992 & Orick & $\mathrm{CA}$ & 5 & 124.059 & 41.287 \\
\hline 4-25-1992 & Piercy & $\mathrm{CA}$ & 5 & 123.794 & 39.966 \\
\hline 4-25-1992 & Salyer & CA & 5 & 123.583 & 40.890 \\
\hline $4-25-1992$ & Trinidad & CA & 5 & 124.142 & 41.059 \\
\hline
\end{tabular}


Table 1. Modified Mercalli Intensity Observations for California North-Coast Earthquakes

\begin{tabular}{|c|c|c|c|c|c|}
\hline Mo-Day-Yr & Location & St & MMI & Long $\left({ }^{\circ} \mathrm{W}\right)$ & $\operatorname{Lat}\left({ }^{\circ} \mathbf{N}\right)$ \\
\hline 4-25-1992 & Westhaven & $\mathrm{CA}$ & 5 & 124.110 & 41.036 \\
\hline $4-25-1992$ & Westport & $\mathrm{CA}$ & 5 & 123.782 & 39.636 \\
\hline 4-25-1992 & Willits & $\mathrm{CA}$ & 5 & 123.354 & 39.410 \\
\hline $4-25-1992$ & Willow Creek & $\mathrm{CA}$ & 5 & 123.630 & 40.940 \\
\hline $4-25-1992$ & Annapolis & $\mathrm{CA}$ & 4 & 123.369 & 38.722 \\
\hline $4-25-1992$ & Biggs & $C A$ & 4 & 121.712 & 39.413 \\
\hline $4-25-1992$ & Castella & $\mathrm{CA}$ & 4 & 122.317 & 41.139 \\
\hline $4-25-1992$ & Corning & CA & 4 & 122.178 & 39.928 \\
\hline $4-25-1992$ & Cottonwood & CA & 4 & 122.280 & 40.386 \\
\hline $4-25-1992$ & Dorris & CA & 4 & 121.917 & 41.968 \\
\hline 4-25-1992 & Dunsmuir & $\mathrm{CA}$ & 4 & 122.271 & 41.208 \\
\hline $4-25-1992$ & Durham & $\mathrm{CA}$ & 4 & 121.799 & 39.646 \\
\hline $4-25-1992$ & Edgewood & $\mathrm{CA}$ & 4 & 122.431 & 41.458 \\
\hline $4-25-1992$ & Elk & $\mathrm{CA}$ & 4 & 123.717 & 39.130 \\
\hline $4-25-1992$ & Flournoy & $\mathrm{CA}$ & 4 & 122.435 & 39.921 \\
\hline $4-25-1992$ & Fort Bragg & $\mathrm{CA}$ & 4 & 123.804 & 39.446 \\
\hline $4-25-1992$ & Gasquet & CA & 4 & 123.968 & 41.846 \\
\hline $4-25-1992$ & Hayfork & CA & 4 & 123.182 & 40.554 \\
\hline $4-25-1992$ & Los Molinos & $\mathrm{CA}$ & 4 & 122.099 & 40.021 \\
\hline $4-25-1992$ & Manchester & $\mathrm{CA}$ & 4 & 123.687 & 38.970 \\
\hline 4-25-1992 & Potter Valley & $\mathrm{CA}$ & 4 & 123.112 & 39.322 \\
\hline $4-25-1992$ & Project City & $\mathrm{CA}$ & 4 & 122.351 & 40.680 \\
\hline 4-25-1992 & Red Bluff & CA & 4 & 122.235 & 40.179 \\
\hline 4-25-1992 & Redding & $\mathrm{CA}$ & 4 & 122.391 & 40.587 \\
\hline 4-25-1992 & Sacramento & CA & 4 & 121.493 & 38.582 \\
\hline $4-25-1992$ & Seiad Valley & $\mathrm{CA}$ & 4 & 123.191 & 41.841 \\
\hline 4-25-1992 & Smith River & CA & 4 & 124.146 & 41.928 \\
\hline 4-25-1992 & Tulelake & $\mathrm{CA}$ & 4 & 121.476 & 41.956 \\
\hline 4-25-1992 & Upper Lake & $\mathrm{CA}$ & 4 & 122.909 & 39.165 \\
\hline 4-25-1992 & Vina & $\mathrm{CA}$ & 4 & 122.053 & 39.933 \\
\hline $4-25-1992$ & Weaverville & $\mathrm{CA}$ & 4 & 122.941 & 40.731 \\
\hline 4-25-1992 & Wildwood & $\mathrm{CA}$ & 4 & 123.053 & 40.400 \\
\hline $4-25-1992$ & Willows & $\mathrm{CA}$ & 4 & 122.193 & 39.524 \\
\hline $4-25-1992$ & Anderson & $\mathrm{CA}$ & 3 & 122.297 & 40.448 \\
\hline 4-25-1992 & Bolinas & $\mathrm{CA}$ & 3 & 122.685 & 37.909 \\
\hline 4-25-1992 & Boonville & $\mathrm{CA}$ & 3 & 123.365 & 39.009 \\
\hline 4-25-1992 & Burney & $\mathrm{CA}$ & 3 & 121.660 & 40.883 \\
\hline 4-25-1992 & Callahan & $\mathrm{CA}$ & 3 & 122.800 & 41.310 \\
\hline 4-25-1992 & Calpella & CA & 3 & 123.203 & 39.234 \\
\hline 4-25-1992 & Carmichael & $\mathrm{CA}$ & 3 & 121.327 & 38.617 \\
\hline $4-25-1992$ & Carson City & NV & 3 & 119.766 & 39.164 \\
\hline $4-25-1992$ & Central Valley & $\mathrm{CA}$ & 3 & 122.370 & 40.681 \\
\hline $4-25-1992$ & Chico & CA & 3 & 121.836 & 39.729 \\
\hline $4-25-1992$ & Cloverdale & $\mathrm{CA}$ & 3 & 123.016 & 38.806 \\
\hline $4-25-1992$ & Dixon & CA & 3 & 121.822 & 38.446 \\
\hline $4-25-1992$ & Fall River Mills & $\mathrm{CA}$ & 3 & 121.437 & 41.005 \\
\hline
\end{tabular}


Table 1. Modified Mercalli Intensity Observations for California North-Coast Earthquakes

\begin{tabular}{|c|c|c|c|c|c|}
\hline Mo-Day-Yr & Location & St & MMI & Long $\left({ }^{\circ} W\right)$ & Lat $\left({ }^{\circ} N\right)$ \\
\hline $4-25-1992$ & Finley & $\mathrm{CA}$ & 3 & 122.874 & 39.004 \\
\hline $4-25-1992$ & Forks Of Salmon & $\mathrm{CA}$ & 3 & 123.330 & 41.260 \\
\hline $4-25-1992$ & Gazelle & $\mathrm{CA}$ & 3 & 122.519 & 41.521 \\
\hline 4-25-1992 & Gerber & $\mathrm{CA}$ & 3 & 122.149 & 40.056 \\
\hline 4-25-1992 & Grass Valley & $\mathrm{CA}$ & 3 & 121.060 & 39.219 \\
\hline $4-25-1992$ & Guerneville & CA & 3 & 122.995 & 38.502 \\
\hline 4-25-1992 & Half Moon Bay & $\mathrm{CA}$ & 3 & 122.428 & 37.464 \\
\hline $4-25-1992$ & Hyampom & CA & 3 & 123.451 & 40.618 \\
\hline 4-25-1992 & Junction City & $\mathrm{CA}$ & 3 & 123.053 & 40.733 \\
\hline 4-25-1992 & Kelseyville & CA & 3 & 122.838 & 38.978 \\
\hline $4-25-1992$ & Magalia & $\mathrm{CA}$ & 3 & 121.577 & 39.812 \\
\hline 4-25-1992 & Mccloud & $\mathrm{CA}$ & 3 & 122.138 & 41.256 \\
\hline 4-25-1992 & Montague & $\mathrm{CA}$ & 3 & 122.527 & 41.728 \\
\hline 4-25-1992 & Nevada City & $\mathrm{CA}$ & 3 & 121.015 & 39.262 \\
\hline 4-25-1992 & Oak Run & CA & 3 & 122.024 & 40.685 \\
\hline 4-25-1992 & Orleans & CA & 3 & 123.540 & 41.302 \\
\hline 4-25-1992 & Palermo & $\mathrm{CA}$ & 3 & 121.537 & 39.436 \\
\hline 4-25-1992 & Palo Cedro & CA & 3 & 122.238 & 40.564 \\
\hline 4-25-1992 & Paskenta & CA & 3 & 122.545 & 39.885 \\
\hline 4-25-1992 & Philo & $\mathrm{CA}$ & 3 & 123.444 & 39.066 \\
\hline 4-25-1992 & Rio Vista & CA & 3 & 121.690 & 38.156 \\
\hline $4-25-1992$ & Shasta & $\mathrm{CA}$ & 3 & 122.491 & 40.599 \\
\hline $4-25-1992$ & Stewarts Point & $\mathrm{CA}$ & 3 & 123.398 & 38.652 \\
\hline $4-25-1992$ & Stonyford & $\mathrm{CA}$ & 3 & 122.543 & 39.375 \\
\hline $4-25-1992$ & Tehama & $\mathrm{CA}$ & 3 & 122.122 & 40.027 \\
\hline $4-25-1992$ & Whiskeytown & CA & 3 & 122.559 & 40.639 \\
\hline 4-25-1992 & Williams & $\mathrm{CA}$ & 3 & 122.148 & 39.155 \\
\hline $4-25-1992$ & Yorkville & $\mathrm{CA}$ & 3 & 123.213 & 38.898 \\
\hline $9-1-1994$ & Honeydew & $\mathrm{CA}$ & 6 & 124.122 & 40.244 \\
\hline $9-1-1994$ & Arcata & CA & 5 & 124.082 & 40.867 \\
\hline $9-1-1994$ & Bayside & CA & 5 & 124.063 & 40.843 \\
\hline $9-1-1994$ & Blocksburg & $\mathrm{CA}$ & 5 & 123.635 & 40.276 \\
\hline $9-1-1994$ & Bridgeville & CA & 5 & 123.799 & 40.469 \\
\hline $9-1-1994$ & Comptche & $\mathrm{CA}$ & 5 & 123.590 & 39.265 \\
\hline $9-1-1994$ & Dos Rios & CA & 5 & 123.352 & 39.717 \\
\hline $9-1-1994$ & Douglas City & CA & 5 & 122.944 & 40.652 \\
\hline 9-1-1994 & Eureka & CA & 5 & 124.163 & 40.802 \\
\hline 9-1-1994 & Ferndale & CA & 5 & 124.263 & 40.576 \\
\hline $9-1-1994$ & Fields Landing & $\mathrm{CA}$ & 5 & 124.214 & 40.725 \\
\hline $9-1-1994$ & Fortuna & CA & 5 & 124.156 & 40.598 \\
\hline $9-1-1994$ & Garberville & CA & 5 & 123.794 & 40.100 \\
\hline $9-1-1994$ & Junction City & $\mathrm{CA}$ & 5 & 123.053 & 40.733 \\
\hline $9-1-1994$ & Lakehead & $\mathrm{CA}$ & 5 & 122.378 & 40.905 \\
\hline $9-1-1994$ & Manchester & CA & 5 & 123.687 & 38.970 \\
\hline $9-1-1994$ & Manila & $\mathrm{CA}$ & 5 & 124.161 & 40.852 \\
\hline $9-1-1994$ & Mckinleyville & CA & 5 & 124.099 & 40.947 \\
\hline
\end{tabular}


Table 1. Modified Mercalli Intensity Observations for California North-Coast Earthquakes

\begin{tabular}{|c|c|c|c|c|c|}
\hline Mo-Day-Yr & Location & St & MMI & Long $\left({ }^{\circ} W\right)$ & Lat $\left({ }^{\circ} \mathbf{N}\right)$ \\
\hline $9-1-1994$ & Mendocino & $\mathrm{CA}$ & 5 & 123.798 & 39.308 \\
\hline $9-1-1994$ & Miranda & CA & 5 & 123.823 & 40.235 \\
\hline $9-1-1994$ & Myers Flat & $\mathrm{CA}$ & 5 & 123.869 & 40.266 \\
\hline $9-1-1994$ & Petrolia & CA & 5 & 124.286 & 40.326 \\
\hline $9-1-1994$ & Phillipsville & $\mathrm{CA}$ & 5 & 123.785 & 40.209 \\
\hline $9-1-1994$ & Piercy & $\mathrm{CA}$ & 5 & 123.794 & 39.966 \\
\hline $9-1-1994$ & Project City & $\mathrm{CA}$ & 5 & 122.351 & 40.680 \\
\hline $9-1-1994$ & Rio Dell & CA & 5 & 124.105 & 40.499 \\
\hline $9-1-1994$ & Samoa & $\mathrm{CA}$ & 5 & 124.185 & 40.819 \\
\hline $9-1-1994$ & Westport & $\mathrm{CA}$ & 5 & 123.782 & 39.636 \\
\hline $9-1-1994$ & Whitethorn & $\mathrm{CA}$ & 5 & 123.942 & 40.024 \\
\hline $9-1-1994$ & Zenia & $\mathrm{CA}$ & 5 & 123.491 & 40.206 \\
\hline $9-1-1994$ & Albion & $\mathrm{CA}$ & 4 & 123.768 & 39.224 \\
\hline $9-1-1994$ & Alderpoint & $\mathrm{CA}$ & 4 & 123.611 & 40.176 \\
\hline $9-1-1994$ & Alturas & $\mathrm{CA}$ & 4 & 120.541 & 41.487 \\
\hline $9-1-1994$ & Annapolis & CA & 4 & 123.369 & 38.722 \\
\hline $9-1-1994$ & Big Bar & $\mathrm{CA}$ & 4 & 123.255 & 40.741 \\
\hline $9-1-1994$ & Blue Lake & $\mathrm{CA}$ & 4 & 123.983 & 40.883 \\
\hline $9-1-1994$ & Branscomb & $\mathrm{CA}$ & 4 & 123.624 & 39.654 \\
\hline $9-1-1994$ & Burnt Ranch & $\mathrm{CA}$ & 4 & 123.473 & 40.809 \\
\hline $9-1-1994$ & Carlotta & CA & 4 & 124.059 & 40.538 \\
\hline $9-1-1994$ & Cave Junction & OR & 4 & 123.647 & 42.163 \\
\hline $9-1-1994$ & Chico & $\mathrm{CA}$ & 4 & 121.836 & 39.729 \\
\hline $9-1-1994$ & Covelo & $\mathrm{CA}$ & 4 & 123.247 & 39.793 \\
\hline $9-1-1994$ & Crescent City & $\mathrm{CA}$ & 4 & 124.201 & 41.756 \\
\hline $9-1-1994$ & Elk & CA & 4 & 123.717 & 39.130 \\
\hline $9-1-1994$ & Enterprise & $\mathrm{CA}$ & 4 & 122.342 & 40.564 \\
\hline $9-1-1994$ & Fort Bragg & $\mathrm{CA}$ & 4 & 123.804 & 39.446 \\
\hline $9-1-1994$ & Freshwater & $\mathrm{CA}$ & 4 & 124.061 & 40.762 \\
\hline $9-1-1994$ & Greenview & $\mathrm{CA}$ & 4 & 122.904 & 41.551 \\
\hline $9-1-1994$ & Hyampom & CA & 4 & 123.451 & 40.618 \\
\hline $9-1-1994$ & Hydesville & $\mathrm{CA}$ & 4 & 124.096 & 40.548 \\
\hline $9-1-1994$ & Kneeland & $\mathrm{CA}$ & 4 & 123.994 & 40.761 \\
\hline $9-1-1994$ & Korbel & $\mathrm{CA}$ & 4 & 123.957 & 40.871 \\
\hline $9-1-1994$ & Laytonville & $\mathrm{CA}$ & 4 & 123.482 & 39.688 \\
\hline $9-1-1994$ & Loleta & $\mathrm{CA}$ & 4 & 124.224 & 40.641 \\
\hline $9-1-1994$ & Medford & OR & 4 & 122.874 & 42.327 \\
\hline $9-1-1994$ & Orick & $\mathrm{CA}$ & 4 & 124.059 & 41.287 \\
\hline $9-1-1994$ & Orleans & $\mathrm{CA}$ & 4 & 123.540 & 41.302 \\
\hline $9-1-1994$ & Patricks Point & $\mathrm{CA}$ & 4 & 124.156 & 41.124 \\
\hline $9-1-1994$ & Pistol River & OR & 4 & 124.394 & 42.277 \\
\hline $9-1-1994$ & Port Orford & CR & 4 & 124.496 & 42.746 \\
\hline $9-1-1994$ & Potter Valley & $\mathrm{CA}$ & 4 & 123.112 & 39.322 \\
\hline $9-1-1994$ & Redcrest & $\mathrm{CA}$ & 4 & 123.949 & 40.401 \\
\hline $9-1-1994$ & Salyer & $\mathrm{CA}$ & 4 & 123.583 & 40.890 \\
\hline $9-1-1994$ & Scotia & $\mathrm{CA}$ & 4 & 124.100 & 40.483 \\
\hline
\end{tabular}


Table 1. Modified Mercalli Intensity Observations for California North-Coast Earthquakes

\begin{tabular}{|c|c|c|c|c|c|}
\hline Mo-Day-Yr & Location & St & MMI & Long( $\left({ }^{\circ} W\right)$ & Lat $\left({ }^{\circ} \mathbf{N}\right)$ \\
\hline $9-1-1994$ & Shasta & $\mathrm{CA}$ & 4 & 122.491 & 40.599 \\
\hline $9-1-1994$ & Trinidad & $\mathrm{CA}$ & 4 & 124.142 & 41.059 \\
\hline $9-1-1994$ & Weott & $\mathrm{CA}$ & 4 & 123.921 & 40.322 \\
\hline $9-1-1994$ & Westhaven & $\mathrm{CA}$ & 4 & 124.110 & 41.036 \\
\hline $9-1-1994$ & Wildwood & $\mathrm{CA}$ & 4 & 123.053 & 40.400 \\
\hline $9-1-1994$ & Willits & $\mathrm{CA}$ & 4 & 123.354 & 39.410 \\
\hline $9-1-1994$ & Willow Creek & $\mathrm{CA}$ & 4 & 123.630 & 40.940 \\
\hline $9-1-1994$ & Auburn & $\mathrm{CA}$ & 3 & 121.076 & 38.897 \\
\hline $9-1-1994$ & Bandon & OR & 3 & 124.410 & 43.120 \\
\hline $9-1-1994$ & Bodega Bay & $\mathrm{CA}$ & 3 & 123.047 & 38.333 \\
\hline $9-1-1994$ & Boonville & $\mathrm{CA}$ & 3 & 123.365 & 39.009 \\
\hline $9-1-1994$ & Brookings & OR & 3 & 124.283 & 42.053 \\
\hline $9-1-1994$ & Burney & CA & 3 & 121.660 & 40.883 \\
\hline 9-1-1994 & Chester & $\mathrm{CA}$ & 3 & 121.231 & 40.306 \\
\hline 9-1-1994 & Chicago Park & $\mathrm{CA}$ & 3 & 120.960 & 39.150 \\
\hline $9-1-1994$ & Cloverdale & $\mathrm{CA}$ & 3 & 123.016 & 38.806 \\
\hline $9-1-1994$ & Cottonwood & $\mathrm{CA}$ & 3 & 122.280 & 40.386 \\
\hline 9-1-1994 & Courtland & $\mathrm{CA}$ & 3 & 121.568 & 38.331 \\
\hline $9-1-1994$ & Dorris & $\mathrm{CA}$ & 3 & 121.917 & 41.968 \\
\hline $9-1-1994$ & Etna & $\mathrm{CA}$ & 3 & 122.894 & 41.457 \\
\hline $9-1-1994$ & Fall River Mills & CA & 3 & 121.437 & 41.005 \\
\hline $9-1-1994$ & Finley & $\mathrm{CA}$ & 3 & 122.874 & 39.004 \\
\hline $9-1-1994$ & Forks Of Salmon & $\mathrm{CA}$ & 3 & 123.330 & 41.260 \\
\hline $9-1-1994$ & Fort Jones & $\mathrm{CA}$ & 3 & 122.839 & 41.608 \\
\hline $9-1-1994$ & French Gulch & $\mathrm{CA}$ & 3 & 122.637 & 40.701 \\
\hline $9-1-1994$ & Fulton & $\mathrm{CA}$ & 3 & 122.769 & 38.496 \\
\hline $9-1-1994$ & Gasquet & $\mathrm{CA}$ & 3 & 123.968 & 41.846 \\
\hline $9-1-1994$ & Glendale & OR & 3 & 123.422 & 42.736 \\
\hline $9-1-1994$ & Gold Beach & OR & 3 & 124.421 & 42.408 \\
\hline $9-1-1994$ & Hayfork & $C A$ & 3 & 123.182 & 40.554 \\
\hline $9-1-1994$ & Hoopa & $\mathrm{CA}$ & 3 & 123.673 & 41.051 \\
\hline $9-1-1994$ & Klamath & $\mathrm{CA}$ & 3 & 124.037 & 41.527 \\
\hline $9-1-1994$ & Klamath Falls & OR & 3 & 121.781 & 42.225 \\
\hline $9-1-1994$ & Lakeport & $\mathrm{CA}$ & 3 & 122.915 & 39.043 \\
\hline $9-1-1994$ & Lewiston & $\mathrm{CA}$ & 3 & 122.806 & 40.708 \\
\hline $9-1-1994$ & Lower Lake & $\mathrm{CA}$ & 3 & 122.609 & 38.911 \\
\hline $9-1-1994$ & Magalia & $\mathrm{CA}$ & 3 & 121.577 & 39.812 \\
\hline $9-1-1994$ & Mount Shasta & $\mathrm{CA}$ & 3 & 122.309 & 41.310 \\
\hline $9-1-1994$ & Mountain View & $\mathrm{CA}$ & 3 & 122.083 & 37.386 \\
\hline $9-1-1994$ & Navarro & $\mathrm{CA}$ & 3 & 123.541 & 39.152 \\
\hline $9-1-1994$ & Nevada City & $\mathrm{CA}$ & 3 & 121.015 & 39.262 \\
\hline $9-1-1994$ & Oroville & $\mathrm{CA}$ & 3 & 121.555 & 39.514 \\
\hline $9-1-1994$ & Palo Cedro & $\mathrm{CA}$ & 3 & 122.238 & 40.564 \\
\hline $9-1-1994$ & Petaluma & $\mathrm{CA}$ & 3 & 122.636 & 38.233 \\
\hline $9-1-1994$ & Platina & $\mathrm{CA}$ & 3 & 122.890 & 40.360 \\
\hline $9-1-1994$ & Point Arena & $\mathrm{CA}$ & 3 & 123.692 & 38.909 \\
\hline
\end{tabular}


Table 1. Modified Mercalli Intensity Observations for California North-Coast Earthquakes

$\begin{array}{llllll}\text { Mo-Day-Yr } & \text { Location } & \text { St } & \text { MMI } & \left.\text { Long( }{ }^{\circ} \mathbf{W}\right) & \left.\text { Lat( }{ }^{\circ} \mathbf{N}\right) \\ 9-1-1994 & \text { Quincy } & \text { CA } & 3 & 120.946 & 39.937 \\ 9-1-1994 & \text { Red Bluff } & \text { CA } & 3 & 122.235 & 40.179 \\ 9-1-1994 & \text { Redding } & \text { CA } & 3 & 122.391 & 40.587 \\ 9-1-1994 & \text { Redwood Valley } & \text { CA } & 3 & 123.203 & 39.266 \\ 9-1-1994 & \text { Roseburg } & \text { CR } & 3 & 123.341 & 43.217 \\ 9-1-1994 & \text { Ruth } & \text { CA } & 3 & 123.320 & 40.270 \\ 9-1-1994 & \text { Saint Helena } & \text { CA } & 3 & 122.469 & 38.505 \\ 9-1-1994 & \text { San Francisco } & \text { CA } & 3 & 122.418 & 37.775 \\ 9-1-1994 & \text { San Leandro } & \text { CA } & 3 & 122.155 & 37.725 \\ 9-1-1994 & \text { Santa Rosa } & \text { CA } & 3 & 122.713 & 38.441 \\ 9-1-1994 & \text { Sebastopol } & \text { CA } & 3 & 122.823 & 38.402 \\ 9-1-1994 & \text { Seiad Valley } & \text { CA } & 3 & 123.191 & 41.841 \\ 9-1-1994 & \text { Selma } & \text { CR } & 3 & 123.615 & 42.279 \\ 9-1-1994 & \text { Smith River } & \text { CA } & 3 & 124.146 & 41.928 \\ 9-1-1994 & \text { Stewarts Point } & \text { CA } & 3 & 123.398 & 38.652 \\ 9-1-1994 & \text { Summit City } & \text { CA } & 3 & 122.401 & 40.686 \\ 9-1-1994 & \text { Ukiah } & \text { CA } & 3 & 123.207 & 39.150 \\ 9-1-1994 & \text { Weaverville } & \text { CA } & 3 & 122.941 & 40.731 \\ 9-1-1994 & \text { Weed } & \text { CA } & 3 & 122.385 & 41.423 \\ 9-1-1994 & \text { Williams } & \text { CA } & 3 & 122.148 & 39.155 \\ 9-1-1994 & \text { Willows } & \text { CA } & 3 & 122.193 & 39.524 \\ 9-1-1994 & \text { Yorkville } & \text { CA } & 3 & 123.213 & 38.898\end{array}$

